TRANSACTIONS OF THE

AMERICAN MATHEMATICAL SOCIETY

Volume 364, Number 10, October 2012, Pages 5525-5569

S 0002-9947(2012)05565-6

Article electronically published on May 24, 2012

\title{
FULL QUIVERS OF REPRESENTATIONS OF ALGEBRAS
}

\author{
ALEXEI BELOV-KANEL, LOUIS H. ROWEN, AND UZI VISHNE
}

\begin{abstract}
We introduce the notion of the full quiver of a representation of an algebra, which is a cover of the (classical) quiver, but which captures properties of the representation itself. Gluing of vertices and of arrows enables one to study subtle combinatorial aspects of algebras which are lost in the classical quiver. Full quivers of representations apply especially well to Zariski closed algebras, which have properties very like those of finite dimensional algebras over fields. By choosing the representation appropriately, one can restrict the gluing to two main types: Frobenius (along the diagonal) and, more generally, proportional Frobenius gluing (above the diagonal), and our main result is that any representable algebra has a faithful representation described completely by such a full quiver. Further reductions are considered, which bear on the polynomial identities.
\end{abstract}

\section{Contents}

1. Introduction

2. Review of Zariski closed algebras

2.1. Polynomial relations of Zariski closed algebras

2.2. The role of Peirce decompositions

3. Full quivers of representations

3.1. Review of classical quivers

3.2. Definition of the full quiver

3.3. Primitive and imprimitive arrows

3.4. Proportional Frobenius gluing (of arrows)

3.5. Synopsis of the full quiver

3.6. The full quiver covers the classical quiver

3.7. Sub-quivers

3.8. Full quivers viewed structurally

4. Specific classes of full quivers

4.1. Full quivers of semisimple algebras

4.2. Elementary full quivers

4.3. Branches of full quivers

5. Gluing, compression of full quivers, and change of base ring

5.1. Compressed full quivers

5.2. Partial gluing up to infinitesimals

5.3. Proportional gluing

5.4. Gluing between branches - Permuted gluing

6. Improving the full quiver

6.1. Irreducible varieties

Received by the editors May 31, 2010 and, in revised form, January 27, 2011.

2010 Mathematics Subject Classification. Primary 16R99, 16G99.

This research was supported by the Israel Science Foundation (grant No. 1178/06). 
6.2. Reduction to proportional Frobenius gluing

7. Decomposing quivers

7.1. Decomposing quivers

7.2. Idempotents and sub-quivers

7.3. Appendix: Pseudo-quivers

\section{INTRODUCTION}

This paper is part of an ongoing project, elaborating upon [3], 6, to lay a firm foundation for Belov's positive solution for Specht's problem for affine algebras with polynomial identity (PI-algebras) in characteristic $p>0$; cf. 3. Briefly, two algebras are called PI-equivalent if they satisfy the same PIs, and the major question in PI-theory is to classify PI-equivalence classes of algebras, thereby classifying varieties. The first question in this direction is Specht's problem, which asks whether every variety is determined by a finite set of PIs. Kemer 20, 21] solved Specht's problem in characteristic 0 for arbitrary algebras, although Specht's problem has counterexamples in characteristic $p$; cf. 2, [16. One of Kemer's main results was that every affine PI-algebra over an infinite field is PI-equivalent to a finite dimensional (f.d.) algebra, and thus combinatoric properties of finite dimensional algebras could be used to finish the solution of Kemer's problem in this case.

In general, we recall [7, pp. 28ff.] that an algebra $A$ over an integral domain $C$ is representable if it can be embedded as a $C$-subalgebra of $\mathrm{M}_{n}(K)$ for a suitable field $K \supset C$ (which can be much larger than $C$ ). Besides their intrinsic importance in many aspects of algebra, representable algebras obviously satisfy the PIs of $n \times n$ matrices, and are the major examples of PI-rings. One of Kemer's theorems states that, over an arbitrary infinite field, every relatively free affine PI-algebra is representable, and thus every variety of PI-algebras contains a representable algebra.

Since the situation for affine PI-algebras over arbitrary fields is more complicated, one is led to search representation theory for useful tools. Ever since the pioneering works of Gabriel [13] and Bernštel̆n-Gelfand-Ponomarev [12, one of the most important such tools has been the quiver of a finite dimensional algebra.

One major difficulty which arises immediately is that quivers are most easily applied to algebras over algebraically closed fields. Serious complications arise for algebras over finite fields. For example, let $\mathbb{F}_{q}$ denote the field of $q$ elements, where $q$ is a power of the prime number $p$. Whereas over an infinite field, any affine PI-algebra is PI-equivalent to a f.d. algebra, this assertion fails for PI-algebras over the finite field $F=\mathbb{F}_{q}$, as exemplified by the algebra $\left(\begin{array}{cc}F & F[\lambda] \\ 0 & F[\lambda]\end{array}\right)$.

In this way, we are led to consider a more detailed combinatoric object, the full quiver of a representation of an algebra, which turns out to be a cover of the usual quiver. Although much of the theory can be formulated for algebras over arbitrary commutative affine rings, we limit our attention mostly to algebras over an (arbitrary) field $R$. 
We focus on Zariski closed algebras, described in [9] and reviewed below. All f.d. algebras are Zariski closed, and Zariski closed algebras satisfy the basic structure theorems that Wedderburn proved for f.d. algebras. In particular, the radical $J$ of a Zariski closed algebra $A$ is nilpotent, and one can write $A=S \oplus J$, where $S$ is a semisimple subalgebra of $A$. The vertices of the full quiver of a representation of $A$ correspond to the diagonal blocks arising from simple components of the semisimple part $S$, whereas the arrows correspond to linear functions between diagonal blocks, and come from the radical $J$.

As noted in [9, Lemma 3.18], any algebra is PI-equivalent to its Zariski closure. Thus, the PI classification problem reduces to classifying Zariski closed algebras of relatively free algebras in terms of PIs. In this paper we study full quivers of representations, with special attention to finding the quiver having the best form; our interest lies in how the full quiver describes the interaction between the radical of an algebra and the semisimple part. The applications to PI-theory are given in [10].

Our study of full quivers is based on the structure of Zariski closed algebras. In [9] we showed that any Zariski closed $F$-subalgebra $A$ of $\mathrm{M}_{n}(K)$ (where $K$ is an algebraically closed field containing $F$ ) has a Wedderburn decomposition in which it is a direct sum of the radical and the semisimple part; furthermore, $A$ can be represented in Wedderburn block form, in which diagonal blocks comprise the semisimple part, and the radical embeds above the diagonal (see Definition 2.10), with certain identifications of the blocks which we call gluing. Gluing occurs separately for the diagonal (semisimple) and the off-diagonal (radical) components.

Diagonal gluing is very easily described, since any identification of diagonal blocks can be viewed as an isomorphism of matrix algebras, which in turn is described in terms of an isomorphism of their fields of scalars. But the automorphisms of finite fields are given by powers of the Frobenius map $a \mapsto a^{p}$, where $p$ is the characteristic, so all diagonal gluing can be described in terms of Frobenius gluing of the diagonal blocks, as illustrated in the following example: When $\operatorname{char}(F)=p$, we have the Frobenius automorphism $\alpha \mapsto \alpha^{p^{u}}$ of $F$, and one can construct the algebra $\left\{\left(\begin{array}{cc}\alpha^{p^{u}} & * \\ 0 & \alpha\end{array}\right): \alpha \in F\right\}$ (which satisfies the PI $\left.x[y, z]-[y, z] x^{p^{u}}\right)$. We also call $p^{u}$ the Frobenius twist in the gluing. When $u=0$, i.e., when this automorphism is the identity, we say there is no Frobenius twist, and call the gluing identical gluing. [9, Theorem 5.14] (given below as Theorem 2.12) describes all gluing along the diagonal blocks.

Thus, our theory reduces to the description of the blocks above the diagonal, in terms of the full quiver. Our overall objective in this paper is to use the full quiver to determine various properties of the underlying algebras, especially the interaction between the semisimple part and the radical. This task is fairly easy when the quiver is straightforward enough. The simplest kind of quiver, consisting of a single arrow and called an elementary quiver, already displays such interactions.

There can also be gluing above the diagonal, i.e., among arrows, which gives rise to relations among the radical elements. We call this off-diagonal gluing. Off-diagonal gluing of two arrows entails gluing of their initial vertices and terminal vertices. For example, one can have identical off-diagonal gluing of two arrows whose initial vertices have Frobenius gluing on the diagonal; this indicates an identification of components via a Frobenius automorphism of the underlying field. 
A certain kind of gluing, called compression, enables us to shrink the size of the representation, at the expense of replacing the base field $F$ by an $F$-algebra with nilpotent elements, which we call infinitesimals.

Another kind of (off-diagonal) gluing is called Frobenius proportional, by which we mean that gluing between two radical components is by means of some scalar multiple (perhaps involving a Frobenius automorphism of the initial or terminal vertices of the arrows). If these Frobenius automorphisms are trivial, we define the gluing to be purely proportional. But one could also have other sorts of gluing which are much more complicated and very difficult to describe. Different representations of the same algebra will produce different full quivers, which on the one hand leads to ambiguity, but also permits us to change the representation to obtain a quiver of a better form.

The main goal of this paper is to obtain the full quiver in the best form. Since the Frobenius twist only occurs for $F$ finite, our theory becomes much easier over infinite fields. To obtain the sharpest possible results, we must limit our attention to the full quiver of relatively free algebras. Towards this end, we have the following theorems:

Theorem 1.1 (cf. Theorem 6.8). The Zariski closure of any representable affine PI-algebra over an infinite field has a representation and full quiver all of whose polynomial relations are consequences of proportional gluing.

Theorem 1.2 (cf. Theorem 6.10). Any relatively free affine PI-algebra over an infinite field has a representation whose quiver has no double edges (between two adjacent vertices) in the full quiver. More generally, any two branches between two vertices are proportionally permuted (see Definition [5.15).

Theorem 1.3 (cf. Theorem 6.12). Any relatively free affine PI-algebra has a representation for whose full quiver all gluing is Frobenius proportional.

We stress that all the polynomial relations of an algebra are consequences of gluing relations of its full quiver. (For example, in Theorem 6.8 they must be consequences of proportional gluing.) Due to interactions of quiver branches for finite fields, in order to obtain the sharpest reformulation of Theorem6.10, we need to introduce gradings in the forthcoming paper [10].

In the appendix, we introduce the notion of pseudo-quivers, to prove the following result:

Theorem 1.4 (cf. Theorem [7.18). Any Zariski closed algebra has a representation for whose full quiver the maximal length of the branches equals the index of nilpotence of the radical minus 1 .

A maximal path in the full quiver is called a branch. Gluing between branches is called total if for each arrow in one branch there is an arrow of the other branch, glued proportionally to it. One candidate for the best form of a full quiver is called indecomposable; cf. Definition 7.4

In a subsequent paper [10, we discuss canonization theorems for full quivers (as well as pseudo-quivers) and apply this theory towards the classification of varieties of PI-algebras, stressing the role of pseudo-quivers. This paper contains the part of the theory concerning the representations of algebras; in [10] we introduce techniques of evaluating polynomials by means of the full quivers, thereby enabling us to further improve full quivers of relatively free algebras. 
This theory thereby enables us to obtain clearer proofs of some results about varieties of PI-rings and also to motivate and unify several interesting examples in PI-theory.

One specific application in [10: We define a parametric set of identities of an algebra $R$ to be a set of identities of the form

$$
\left\{\sum \xi_{j} f_{j}: \xi_{j} \in F\right\}
$$

where the $f_{j}$ are homogeneous polynomials that are not identities of $R$.

Branches in the quiver, together with gluing, often define a parametric set of PIs, although these identities are not always definable over the base field $F$ but sometimes require passing to a transcendental field extension of $F$. Other identities arise from gluing among different paths of the canonical quiver.

In another direction, one can use quivers to study ring-theoretic properties of relatively free PI-algebras; for example, in [10] we prove the following result:

Theorem. A relatively free affine algebra $A$ is weakly Noetherian iff it has a faithful representation for which each connected component of the full quiver consists of a single arrow, with each vertex having matrix degree at most 1.

\section{Review of Zariski Closed ALGEBRAS}

In this section we review Zariski closed algebras, studied in 9, which form the foundation for the study of representable PI-rings. Suppose $F \subseteq K$ is an algebraically closed field. Let $\rho: A \rightarrow \mathrm{M}_{n}(K)$ be a representation of a ring $A$. The Zariski closure $\rho(A)^{\mathrm{cl}}$ is the closure of $\rho(A)$ with respect to the Zariski topology of $\mathrm{M}_{n}(K)$.

This definition clearly depends on the choice of the representation $\rho$. Nevertheless, abusing language slightly, we assume that $\rho$ is a faithful representation, taken as given, and thus we view the ring $A$ as contained in $\mathrm{M}_{n}(K)$; we call $\rho(A)^{\mathrm{cl}}$ the Zariski closure of $A$, denoted as $A^{\mathrm{cl}}$. For convenience, we assume that our representable ring $A$ is an $F$-algebra.

Lemma 2.1. The radical $J$ of the Zariski closure of any affine PI-algebra $A_{0}$ is nilpotent.

Proof. Let $J_{0}$ be the radical of $A_{0}$. By the Braun-Kemer-Razmyslov theorem, $J_{0}^{\ell}=0$ for some $\ell$. Hence $x_{1} \cdots x_{\ell}$ is an identity of $J_{0}$, and thus of its Zariski closure, which is $J$ by [9, Proposition 3.21]. In other words, $J^{\ell}=0$.

Since any $K$-subalgebra of $\mathrm{M}_{n}(K)$ is Zariski closed, we can take the Zariski closure of $A$ in any $K$-subalgebra $B \subseteq \mathrm{M}_{n}(K)$ that contains $\rho(A)$. In particular, we can take $B$ to be the linear closure $K A$, the $K$-subspace of $\mathrm{M}_{n}(K)$ spanned by $A$.

When $F$ is infinite, the Zariski closure is just the linear closure. Thus, our special interest in the Zariski closure is for algebras over finite fields. As shown in 9], Zariski closed algebras satisfy many of the structural properties of finite dimensional algebras over algebraically closed fields.

Remark 2.2. By [9, Proposition 3.20], we may assume that every ideal of $B=K A$ intersects $A$ nontrivially. These assumptions are implicit in the rest of the text. This ties the structure of $A$ to the structure of $B$. 
Viewing $A \subseteq B$ explicitly as in Remark 2.2 we fix a base $v_{1}, \ldots, v_{m}$ of $B$ over $K$, where each $v_{i} \in A$. Although $A$ need not be a $K$-algebra, every element of $A$ is written as $\alpha_{1} v_{1}+\cdots+\alpha_{m} v_{m}$ for suitable $\alpha_{i} \in K$. In order to study the coefficients, we study their set $\operatorname{poly}(A)$ of polynomial relations, which are defined as those polynomials $f \in K\left[\lambda_{1}, \ldots, \lambda_{m}\right]$ such that $f\left(\alpha_{1}, \ldots, \alpha_{m}\right)=0$ for every $\alpha_{1} v_{1}+\cdots+\alpha_{m} v_{m} \in A$. In particular, polynomial relations must have constant term 0. One advantage of this point of view is that an algebra is Zariski closed if and only if its polynomial relations serve as its defining relations. On the other hand, linear transformations are continuous in the Zariski topology, implying that the Zariski closure is independent of the choice of base of $B$.

One well-known connection to PI-theory is that, when we designate a given base $B$, any PI of the algebra $A$ can be viewed as a set of polynomial relations in the coefficients of the elements of $A$, written in terms of the base $B$; cf. [9, Proposition 3.17]. Hence the Zariski closure $A^{\mathrm{cl}}$ of $A$ is an algebra, which is PI-equivalent to $A$, and we usually take our algebra $A$ to be Zariski closed.

\section{Example 2.3.}

(i) The Zariski closed $F$-subalgebras of an algebraically closed field $K$ are precisely the finite intermediate subfields $F \subseteq F_{1}$ of $K$, as well as $K$ itself. Indeed, any infinite field cannot have nontrivial polynomial relations, and a finite field $F_{1}$ of $q$ elements satisfies the polynomial identity $\lambda_{1}^{q}-\lambda_{1}$. But $F_{1}{ }^{\mathrm{cl}}$ must also satisfy the identity $\lambda_{1}^{q}-\lambda_{1}$ and thus have at most $q$ elements; hence, $F_{1}{ }^{\mathrm{cl}}=F_{1}$.

(ii) Other examples of commutative Zariski closed subalgebras of $\mathrm{M}_{n}(K)$ include

$$
\left\{\left(\begin{array}{ccc}
\alpha & \beta & \gamma \\
0 & \alpha & \beta \\
0 & 0 & \alpha
\end{array}\right): \alpha, \beta, \gamma \in K\right\} \cong K[\lambda] /\left\langle\lambda^{3}\right\rangle .
$$

The starting point in the representation theory of finite dimensional algebras may well be Wedderburn's Principal Theorem.

Theorem 2.4 (Wedderburn's Principal Theorem). If $A$ is a f.d. algebra over an algebraically closed field $K$, then $A$ has a Wedderburn decomposition $A=S \oplus J$, where $J=\operatorname{Rad}(A)$ and $S \cong A / J$ is a subalgebra of $A$ that is a direct sum of matrix algebras over $K$.

The following theorem, parallel to Wedderburn's Principal Theorem, gives us the basic structure of Zariski closed algebras over arbitrary fields.

Theorem 2.5 (First Representation Theorem, [9, Theorem 3.33]). Let $A$ be a representable F-algebra. If $A=A^{\mathrm{cl}}$, then $A$ has a Wedderburn decomposition $A=S \oplus J$, where $J=\operatorname{Rad}(A)$ and $S \cong A / J$ is a subalgebra of $A$ that is isomorphic to a direct sum of matrix algebras over fields (which are closed $F$-subfields of $K$ ).

Thus, the basic question in studying these algebras is determining how the radical $J$ interacts with the semisimple part $S \cong A / J$.

2.1. Polynomial relations of Zariski closed algebras. We review some of the results about polynomial relations of Zariski closed algebras proved in [9].

Definition 2.6. A polynomial of the form $\sum_{i=1}^{m} \sum_{j \geq 1} c_{i j} \lambda_{i}^{q_{i j}}$ is called a $q$-polynomial if each $q_{i j}$ is a $q$-power with $q=|F|$, where we take $q=1$ if $F$ is infinite. 
In [19, 23], 26] it is shown that every additively closed subvariety of affine spaces is given by a system of equations each of which is a $q$-polynomial. In other words, we have the following assertion, which can also be seen by combining Proposition 4.7 and Theorem 4.10 of [9]:

Corollary 2.7. $\operatorname{poly}(A)$ is a finitely generated $F[\phi]$-module (where $\phi$ is the Frobenius map $\left.\lambda_{i} \mapsto \lambda_{i}^{q}\right)$. It has a finite set of defining relations, and every polynomial relation is a consequence of these.

Definition 2.8. A polynomial relation is of F-Frobenius type if it has one of the following three forms, where $q$ is as in the previous definition:

(i) $\lambda_{i}=0$,

(ii) $\lambda_{i}=\lambda_{i}^{s}$, where $s$ is a $q$-power, or

(iii) $\lambda_{i}=\lambda_{j}^{s}, j \neq i$, where $s$ is a $q$-power.

We quote [9, Theorem 4.15]:

Theorem 2.9. Suppose $A$ is a commutative, semiprime Zariski closed $F$-subalgebra of a finite dimensional commutative $K$-algebra $B$. Then $\operatorname{poly}(A)$ is generated by finitely many polynomial relations of F-Frobenius type.

This theorem is applicable to the center of $A$, since the center of a Zariski closed algebra is Zariski closed, by [9, Lemma 3.28]. The next step is to use this description to build a suitable representation of $A$. For this, we also recall (and slightly generalize) a definition from 9 .

Definition 2.10. Let $F \subseteq K$ be commutative Noetherian rings. Suppose $A$ is a $K$-algebra with an ideal $J_{0} \subseteq \operatorname{Jac}(A)$ (the radical of $A$ ), such that

$$
A / J_{0}=A_{1} \times \cdots \times A_{k} \cong \mathrm{M}_{n_{1}}\left(F_{1}\right) \times \cdots \times \mathrm{M}_{n_{t}}\left(F_{k}\right),
$$

for subrings $F \subseteq F_{u} \subseteq K$.

We say that a representation $\rho: A \rightarrow \mathrm{M}_{n}(K)$ is in Wedderburn block form if, for suitable $s_{1}, \ldots, s_{k} \geq 1$, the diagonal of $\mathrm{M}_{n}(K)$ is contained in $s_{1}+\cdots+s_{k}$ disjoint diagonal blocks $A_{u}^{(1)}, \ldots, A_{u}^{\left(s_{u}\right)}(u=1, \ldots, k)$, satisfying the following properties:

- For each $u, A_{u}^{(i)}$ has size $n_{u} \times n_{u}$ for each $i$, and is isomorphic to $A_{u}$.

- $n=\sum_{u} s_{u} n_{u}$.

- The given representation $\rho$ restricts to an embedding $\rho_{u}: A_{u} \rightarrow A_{u}^{(1)} \times \cdots \times$ $A_{u}^{\left(s_{u}\right)}$.

- The ensuing composite of $\rho_{u}$ with the projection onto the $i$ component yields an isomorphism $\rho_{u}^{(i)}: A_{u} \rightarrow A_{u}^{(i)}$ for each $i$.

- $\rho$ embeds $J$ into the sum of the strictly upper triangular blocks (above the diagonal blocks).

For each $u$, the blocks $A_{u}^{(1)}, \ldots, A_{u}^{\left(s_{u}\right)}$ are said to be diagonally glued.

We usually assume that $F$ is a field whose algebraic closure is $K$, and in this case, $J_{0}=\operatorname{Jac}(A)$.

Thus, each of $A_{u}^{(1)}, \ldots, A_{u}^{\left(s_{u}\right)}$ has center isomorphic to $F_{u}$. Using idempotents, when $A$ is Zariski closed (with $F \subseteq K$ fields) we can apply Example 2.3 to conclude that the $F_{u}$ are either finite or equal to $K$. If $F_{u}$ is finite, we say that the corresponding blocks have finite type. 
For example, suppose $F=\mathbb{F}_{q}$. Then

$$
A=\left\{\left(\begin{array}{ccc}
\alpha & 0 & \gamma \\
0 & \beta & \delta \\
0 & 0 & \beta^{q}
\end{array}\right): \quad \alpha, \gamma, \delta \in K, \beta \in \mathbb{F}_{q^{2}}\right\}
$$

is a Zariski closed $F$-subalgebra of $\mathrm{M}_{3}(K)$, defined by the polynomial relations $\lambda_{12}=\lambda_{21}=\lambda_{31}=\lambda_{32}=0$ and $\lambda_{33}-\lambda_{22}^{q}=\lambda_{33}-\lambda_{33}^{q^{2}}=0$. (The last two polynomial relations formally imply the polynomial relation $\lambda_{22}-\lambda_{22}^{q^{2}}=0$.) In this case $A / J \cong K \oplus \mathbb{F}_{q^{2}}$, where the second component, which is finite, embeds via $\beta \mapsto\left(\beta, \beta^{q}\right)$, thus gluing the $e_{22}$ and $e_{33}$ entries.

Here is an easy but important special case.

Remark 2.11. Assume $A \cong A_{1} \times \cdots \times A_{k}$ is semisimple; i.e., $J=0$. Then the only Wedderburn blocks are along the diagonal. Any representation of $A$ restricts to a representation of the center, whose polynomial relations have already been described in Theorem 2.9. These polynomial relations extend to the respective entries of the matrix algebra components, so we conclude that all gluing is diagonal, and is either identical gluing or Frobenius gluing.

For arbitrary Zariski closed algebras, we start with Remark 2.11, since the semisimple part is a subalgebra, but also may have $J \neq 0$, in which case we also need to contend with blocks above the diagonal. The following result from 9 , Theorem 5.14] describes the relations on the diagonal.

Theorem 2.12. Suppose $A \subseteq \mathrm{M}_{n}(K)$ is a Zariski closed algebra, with

$$
A / J=A_{1} \times \cdots \times A_{k},
$$

a direct product of $k$ simple components. Then we can choose the matrix units of $\mathrm{M}_{n}(K)$ in such a way that $A$ has Wedderburn block form, and all identifications among the diagonal blocks are Frobenius gluing.

Recalling the linear closure $B=K A$, we write the diagonal components of $B$ as $B_{1}, B_{2}, \ldots, B_{\ell}$ (taken in $\mathrm{M}_{n}(K)$ ). Letting $e_{r}$ denote the unit element for the component $B_{r}$, for $1 \leq r \leq \ell$, we have $B_{r}=e_{r} B e_{r}$; we also write $B_{r, r^{\prime}}$ for $e_{r} B e_{r^{\prime}}$. (Thus, $B_{r, r}=B_{r}$.) Then

$$
B=\bigoplus_{r, r^{\prime}=1}^{k} B_{r, r^{\prime}}
$$

We carry this notation throughout.

The relations above the diagonal are subtler. It is particularly useful to describe the Wedderburn block form in terms of polynomial relations. Taking the matrix units as a base for $\mathrm{M}_{n}(K)$, the relations module poly $(A)$ of $A$ in Wedderburn block form can be decomposed as a direct sum, in accordance with the decomposition $A=S \oplus J$. Thus $A$ is defined by three classes of relations on the components $A_{r, r^{\prime}}=A \cap B_{r, r^{\prime}}$. (Here we write $A_{r}$ for the block $A_{r, r}$; this would correspond to some $A_{u}^{(i)}$ in the notation of Definition 2.10])

(1) $\lambda_{i j}=0$ for $i>j$ (which says that all entries below the diagonal blocks are $0)$.

(2) There are relations of the form $\lambda_{i j}=\lambda_{i^{\prime} j^{\prime}}^{q^{t}}$, where $(i, j)$ and $\left(i^{\prime}, j^{\prime}\right)$ are in the same relative position in glued blocks; we call this Frobenius gluing 
of exponent $t$. When diagonal blocks $A_{r}$ and $A_{r^{\prime}}$ have Frobenius gluing of exponent $t$, we write $t=\exp \left(A_{r, r^{\prime}}\right)$, and call it the relative Frobenius exponent. We permit $t=0$; in fact we must have $t=0$ when the base field $F$ is infinite. For $F$ finite, $\exp \left(A_{r, r^{\prime}}\right)$ is only well defined modulo the dimension of $F_{r}=F_{r^{\prime}}$ over $F$.

(3) Other relations link components above the diagonal blocks by means of $q$-polynomials.

Note 2.13. Occasionally, we want to deal with algebras without 1 , so let us consider this case briefly. Any finite dimensional algebra $B$ without 1 has a maximal set of orthogonal primitive idempotents $\left\{e_{1}, \ldots, e_{k}\right\}$, whose sum we denote as $e$. We formally define the operator $e_{0}$ by $e_{0} b=b-e b$ and $b e_{0}=b-b e$. Clearly the operator $e_{0}$ is idempotent, and $e_{0} b=0$ iff $b \in e R . B_{0,0}=e_{0} B e_{0}$ must be nilpotent, since otherwise it would contain a nonzero idempotent of $B$, contrary to hypothesis. Strictly speaking, $B_{0}$ may be composed of several nilpotent blocks, as in the example

$$
B=\left\{\left(\begin{array}{llll}
0 & * & 0 & 0 \\
0 & 0 & 0 & 0 \\
0 & 0 & 0 & * \\
0 & 0 & 0 & 0
\end{array}\right)\right\}
$$

Using this Peirce decomposition, one can obtain a corresponding Wedderburn block decomposition, but where one of the diagonal blocks is $B_{0,0}$. The zero block can have arbitrary dimension.

For example, when $B$ is nilpotent, the idempotent $e=0$ and $e_{0}$ is the identity operator and $e_{0} B e_{0}=B$. For

$$
B=\left\{\left(\begin{array}{lll}
0 & 0 & * \\
0 & 0 & * \\
0 & 0 & *
\end{array}\right)\right\}
$$

the block $B_{0}$ is a $2 \times 2$ zero block.

We would like to focus on one particular kind of gluing of nondiagonal blocks.

Definition 2.14. Proportional Frobenius gluing is the situation in which, for suitable powers $q, q^{\prime}$ of $p$, the $q$ power of the entries in $B_{r^{\prime}, s^{\prime}}$ each are $\nu$ times the $q^{\prime}$ power of the respective entries in $B_{r, s}$. When $q, q^{\prime}=1$, this is merely called proportional gluing. When moreover $\nu=1$, we say that the component has identical gluing.

Note that proportional Frobenius gluing is transitive. Other relations of class (3) often are far more complicated to describe than those of classes (11) and (2). Fortunately, we may bypass them, as we shall see.

Definition 2.15. We define the glue equivalence relation on the diagonal blocks, by saying $B_{r} \sim B_{r^{\prime}}$ when these two diagonal blocks are glued. Thus, for $k$ as in Definition 2.10, there are $k$ equivalence classes of glued diagonal blocks, which we call glued components.

Remark 2.16. The relative Frobenius exponents are used to define equivalence relations on vectors of glued indices as follows: Let $T_{u}$ denote the set of those indices $r \in\{1, \ldots, n\}$ such that the diagonal block $B_{r}$ belongs to the $u$ glued component. 
For every $1 \leq r, s \leq k$, we let $T_{u, v}=\left\{(r, s) \in T_{u} \times T_{v}: r \leq s\right\}$, and define an equivalence relation on $T_{u, v}$ by setting $(r, s) \sim\left(r^{\prime}, s^{\prime}\right)$ iff $\exp \left(B_{r, r^{\prime}}\right) \equiv \exp \left(B_{s, s^{\prime}}\right)$ modulo $\operatorname{gcd}\left(t_{r}, t_{s}\right)$, where $t_{r}$ is the dimension of $\operatorname{Cent}\left(B_{r}\right)$.

(Formally we have a partial matrix $\left(\ell_{r s}\right)$, defined when $B_{r}$ and $B_{s}$ are glued, such that $\ell_{r r}=\ell_{s s}$ is the dimension of $F_{r}$ over $F$, and $\ell_{r t}=\ell_{r s}+\ell_{s t}\left(\bmod \ell_{r r} \mathbb{Z}\right)$ if $B_{r}, B_{s}$ and $B_{t}$ are glued.)

\subsection{The role of Peirce decompositions.}

Remark 2.17. One of the primary tools in the analysis of a Zariski closed algebra $A$ is the Peirce decomposition, which we use here at three levels.

- We start with the Peirce decomposition of $A$. The gluing equivalence does not recapture the Wedderburn principal decomposition of Theorem 2.5 in Example 2.3, all the $e_{r}$ are glued, and their sum is the unit element 1. In general, a local algebra has no idempotents, so we cannot extract its semisimple part from its Peirce decomposition!

- The next refinement is the Peirce decomposition of the linear closure $B=$ $K A$. We call this the sub-Peirce decomposition of $A$. Note that nonidentity Frobenius gluing in $A$ becomes "unglued" in $B$, when viewed as an algebra over the infinite field $K$.

- Finally, each idempotent $e$ of $B$ corresponds to a glued component, and we can write $e$ as a sum of unit elements of the glued blocks of this component. This is the finest Peirce decomposition with which we work.

(Other decompositions which come up, especially in the proof of Theorem 6.12, arise from Frobenius gluing, and from algebraic group decompositions.)

Remark 2.18. The Peirce decomposition of an algebra $A$ can be viewed structurally, in terms of decomposing $A=\sum A e_{i}$ as a direct sum of projective modules; then

$$
A \approx \operatorname{End}_{A} A=\operatorname{End}_{A}\left(\sum A e_{i}\right) \cong \bigoplus \operatorname{Hom}_{A}\left(A e_{i}, A e_{j}\right) \cong \bigoplus e_{i} A e_{j} .
$$

Since $A \subset B \subset \mathrm{M}_{n}(K)$ in Remark 2.17, this observation can be applied to $A$ as a subalgebra of any of these three algebras, and $A \cap \operatorname{Hom}\left(\mathrm{M}_{n}(K) e_{i}, \mathrm{M}_{n}(K) e_{j}\right)$ is contained in the direct sum of suitable Wedderburn blocks. Note that each Wedderburn block is an $\mathrm{M}_{n_{i}}\left(F_{i}\right)-\mathrm{M}_{n_{j}}\left(F_{j}\right)$-bimodule in general.

Example 2.19. We can recover the decomposition $A=S \oplus J$ of Theorem 2.5 from the intersection of $A$ with the Wedderburn block components of $B=K A$. In other words, matching components yields

$$
\begin{aligned}
& S=\left(\bigoplus e_{r} B e_{r}\right) \cap A=\left(\bigoplus e_{r} A e_{r}\right) \cap A ; \\
& J=\left(\bigoplus_{r<s} e_{r} B e_{s}\right) \cap A=\left(\bigoplus_{r<s} e_{r} A e_{s}\right) \cap A .
\end{aligned}
$$

\section{Full Quivers of Representations}

We now turn to our main objective, which is to describe representations of algebras in terms of quivers (i.e., directed graphs). First we review the classical theory of quivers; an accessible reference is [1]. Then we introduce our more detailed version, called the full quiver of a representation, whose description relies on Frobenius gluing, and give some examples of how one builds the full quiver from a specific algebra. 
In the subsequent sections we study full quivers in increasing complexity, starting with single vertices and then single arrows (which we call elementary quivers) and next branches, with the aim of interpreting properties of Zariski closed algebras in terms of their full quivers of representations. In the subsequent paper, 10, we distinguish among different kinds of full quivers by means of PIs.

\subsection{Review of classical quivers.}

Definition 3.1. Suppose $\left\{e_{1}, \ldots, e_{t}\right\}$ is a 1-sum set of orthogonal primitive idempotents of a finite dimensional algebra $A$ over a field $F$. The (classical) quiver of $A$ is the graph whose vertices are $\left\{e_{1}, \ldots, e_{t}\right\}$ and whose arrows from $e_{i}$ to $e_{j}$ have multiplicities $\left[e_{i} \operatorname{Rad}(A) e_{j}: F\right]$.

The quiver is independent of the choice of $\left\{e_{1}, \ldots, e_{t}\right\}$, in view of the well-known identification of $e A e$ with $\operatorname{Hom}_{A}(A e, A e)$ and the Krull-Schmidt Theorem.

Example 3.2. (i) $A=F[x] /\left\langle x^{2}\right\rangle$. The quiver is the loop:

(ii) $A=F[x] /\left\langle x^{3}\right\rangle$. The quiver is the double loop:

(iii) $A=\left\{\left(\begin{array}{ll}* & * \\ 0 & *\end{array}\right)\right\} \subset \mathrm{M}_{2}(F)$. The quiver is the graph comprised of a single arrow connecting two vertices:

When considering quivers, one usually passes to $A / J^{2}$, where $J$ is the radical of $A$; then there is the celebrated correspondence between indecomposable modules and Dynkin diagrams, [12, [13], [17], 18]. Of course, in passing to $A / J^{2}$ one forfeits much of the combinatoric structure involving the radical.

Also, one customarily considers the "basic algebra" $\operatorname{End}_{A} P$ Morita equivalent to $A$, for a suitable projective module $P$. These reduced classical quivers, although a powerful tool in much of representation theory, do not help us very much in combinatoric questions such as determining PIs of an algebra.

3.2. Definition of the full quiver. Having established the notion of Zariski closed algebras, we would like to study their structure through the techniques of representations of finite dimensional algebras, by refining the notion of quiver from Definition 3.1. Since we want to study Zariski closed algebras, and these are defined in terms of representations, we need our notion of quiver to be compatible with the particular representation.

Since Zariski closed algebras are semilocal, i.e., $S=A / J$ is semisimple and $J$ is nilpotent, let us begin in the somewhat greater generality of semilocal rings. Writing $S=\prod_{i=1}^{k} \mathrm{M}_{n_{i}}\left(D_{i}\right)$, where $D_{i}$ are division rings with multiplicative unit $\bar{e}_{i}$, we can lift the orthogonal idempotents $\bar{e}_{1}, \ldots, \bar{e}_{k}$ to a set of orthogonal idempotents $e_{1}, \ldots, e_{k}$ of $A$ whose sum is 1 .

The fact that $A=S \oplus J$ enables us to start with the quiver of $S$ as a sub-quiver of that of $A$. We treat these parts in different ways. The loops of the classical quiver could be viewed as ring injections inside $S$, which in turn could be broken down into homomorphisms $D_{i} \rightarrow D_{i^{\prime}}$, and the arrows that are not loops are separated to arrows each of multiplicity 1 . 
Unfortunately, it can be quite difficult to describe all homomorphisms between two given division rings. But in the case under consideration, of a Zariski closed $F$-subalgebra $A$ of $\mathrm{M}_{n}(K)$, the center of each $D_{i}$ is either a finite field or an algebraically closed field, so each $D_{i}$ is a field. Thus, our injections are either the identity map or are given by powers of Frobenius homomorphisms $a \mapsto a^{p}$. In light of Definition 2.10. which involves partitioning the blocks into glued components, our representation is determined by the following:

Definition 3.3. Suppose we are given a representation $\rho: A \rightarrow \mathrm{M}_{n}(K)$, where $A$ is Zariski closed and $\rho(A)$ is in Wedderburn block form, with the blocks $B_{1}, \ldots, B_{\ell}$ along the diagonal.

(1) The full quiver of the representation $\rho$ (of $A$ ) is a directed graph on $k$ vertices corresponding to the diagonal blocks $B_{r}, 1 \leq r \leq \ell$. Each vertex is labelled with a roman numeral $\mathbf{i}$ (taking the values $I, I I$, etc.); vertices corresponding to glued diagonal blocks are labelled with the same roman numeral. The label • denotes a vertex which is not glued. In an algebra without unit, the label $\circ$ indicates a zero diagonal block.

(2) Each vertex is assigned a pair of subscripts $\left(n_{r}, t_{r}\right)$, written as $\bullet_{n_{r}\left(t_{r}\right)}$, where $\left|F_{r}\right|=|F|^{t_{r}}$. The first subscript $n_{r}$ is the degree of the corresponding matrix algebra $B_{r}=\mathrm{M}_{n_{r}}\left(F_{r}\right)$, and is called the matrix degree of the vertex; $\sum_{r=1}^{k} n_{r}=n$. We may suppress the first subscript when $n_{r}=1$.

(3) When $F_{r}=\operatorname{Cent}\left(B_{r}\right)$ is a finite field, the second subscript $t_{r}$ denotes that the cardinality of $F_{r}$ is $|F|^{t_{r}}$; i.e., $\left[F_{r}: F\right]=t_{r}$. In this case the vertex is called finite. Otherwise, the second subscript is left out, which indicates that $F_{r}=K$; then the vertex is called infinite. (When $A$ is Zariski closed, $F_{r}$ is either finite or equal to $K$ as explained above.)

We simplify the notation a bit, by means of the observation that glued diagonal blocks must have the same pairs of subscripts, so we only notate this for the first block in a given glued diagonal component.

(4) When $F$ is a finite field, say of order $q$, superscripts $u \leq t$ indicate the power $\phi^{u}$ of the Frobenius homomorphism $\phi: x \mapsto x^{q}$; the notation $I^{(u)}$ and $I^{\left(\ell^{\prime}\right)}$ indicates blocks belonging to the same glued component, with the identification given by $\phi^{u^{\prime}-u}$. The index $u^{\prime}-u$ is well-defined modulo the corresponding dimension $t_{r}$ of $F_{r}$ over $F$. Absence of superscripts should be read as $u=u^{\prime}=0$; namely, identity gluing.

(5) The full quiver has an arrow (i.e., a directed edge) from $\mathbf{i}_{r}$ to $\mathbf{i}_{s}$ iff $e_{r} \rho(A) e_{s}$ $\neq 0$, where $e_{r}, e_{s}$ are the idempotents corresponding to the vertices. Loops are omitted. Arrows with identical gluing are labelled with the same Greek letter.

(6) Proportional off-diagonal gluing (in other words, the entries in $B_{r^{\prime}, s^{\prime}}$ each are $\nu$ times the respective entries in $B_{r, s}$ ) is indicated by writing some Greek letter (say $\alpha$ ) for the arrow from the $r$ vertex to the $s$ vertex, and writing $\nu \alpha$ for the arrow from the $r^{\prime}$ vertex to the $s^{\prime}$ vertex.

Proportional Frobenius gluing is indicated by writing $\alpha^{q^{u}}$ for the arrow from the $r$ vertex to the $s$ vertex, and writing $\nu \alpha^{q^{u^{\prime}}}$ for the arrow from the $r^{\prime}$ vertex to the $s^{\prime}$ vertex. (We do not write $\nu$ when $\nu=1$.) 
Since the representation is upper diagonal, there can be no cycles. In Theorem 6.8 we show when $F$ is infinite that the representation can be chosen so that its full quiver has only proportional gluing.

Remark 3.4. Conversely, there is an algebra (provided with a representation) corresponding to any full quiver, which can be constructed using path algebras.

Remark 3.5. Any gluing applies in the same way to each entry in the matrix components (and thus corresponds to a direct sum of projective modules). Thus, shrinking the size of all blocks in a glued component to 1 yields a Morita equivalent algebra.

Remark 3.6. For any algebra $A=A_{1} \times \cdots \times A_{t}$, taking the representation of $A$ that is the direct sum of the representations of the $A_{i}$, we see that the full quiver of this representation is the disjoint union of the full quivers of the representations of the $A_{i}$.

We call an algebra indecomposable if it cannot be written as a direct sum of proper subalgebras. Thus, when there is no gluing, the full quiver of a representation is connected (as an undirected graph) iff the corresponding algebra is indecomposable.

We say that two connected components of the full quiver are glued if they have some glued vertices. For example, the full quiver

$$
I \rightarrow I I, I I \rightarrow I I I
$$

is disconnected, but its components are glued by the vertices labeled II. Extending this relation via transitivity, we see that any representation factors into a direct product of its glue-connected components; consequently, we focus on glue-connected full quivers.

Example 3.7. An algebra may have different representations in Wedderburn block form, of the same dimension, which are not conjugate to each other. At times, this phenomenon is related to arrows between glued vertices, as illustrated by the two representations below.
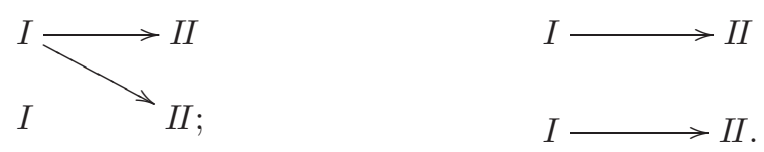

Proposition 3.8. If $\Gamma$ is the full quiver of a representation of an algebra $A$, then the full quiver $\Gamma^{\prime}$ obtained by reversing all arrows in $\Gamma$ is the full quiver of the representation of the opposite algebra $A^{\mathrm{op}}$.

Proof. $\Gamma^{\prime}$ could be viewed as the full quiver of the transpose of the representation (where we permit blocks beneath the diagonal, rather than above it), and this is a transpose representation of $A$, which is isomorphic to the opposite algebra of $A$.

3.3. Primitive and imprimitive arrows. We streamline the full quiver a bit, to ease the notation.

Definition 3.9. An arrow $\mathbf{i} \rightarrow \mathbf{j}$ is called primitive if there is no $\mathbf{k}$ such that there exist arrows $\mathbf{i} \rightarrow \mathbf{k}$ and $\mathbf{k} \rightarrow \mathbf{j}$. We erase all nonprimitive arrows involved in unglued vertices, since these are formal consequences of the primitive arrows, corresponding 
to the product of the corresponding respective elements of the algebra. Thus, we write

$$
I \rightarrow I I \rightarrow I I I \quad \text { or } \quad \bullet \rightarrow \bullet \rightarrow \bullet
$$

for the full quiver of the algebra of upper triangular $3 \times 3$ matrices, although technically there should be an arrow from $I$ to $I I I$. (Gluing of arrows can upset this reasoning, as indicated in Example 5.13 below; therefore glued nonprimitive arrows will not be erased.)

Example 3.10. (1) The algebra

$$
A=\left\{\left(\begin{array}{cccc}
\alpha & * & * & * \\
0 & \beta & \gamma & * \\
0 & 0 & \alpha & * \\
0 & 0 & 0 & \beta^{q}
\end{array}\right): \alpha \in \mathrm{M}_{2}\left(\mathbb{F}_{q^{3}}\right), \beta \in K, \gamma \in \mathrm{M}_{1,2}(K)\right\}
$$

over $F=\mathbb{F}_{q}$, where $K \supseteq \mathbb{F}_{q^{3}}$, has the following full quiver:

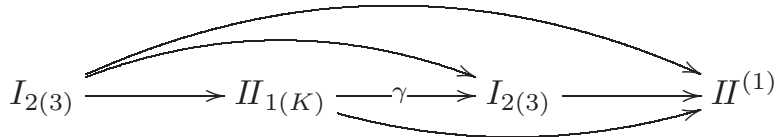

or just the path

$$
I_{2(3)} \longrightarrow I I \longrightarrow I \longrightarrow I^{(1)},
$$

in view of our shorthand conventions and erasing the nonprimitive arrows.

The representation written out in full, in $\mathrm{M}_{6}(K)$, is

$$
\left\{\left(\begin{array}{cccccc}
\alpha_{11} & \alpha_{12} & * & * & * & * \\
\alpha_{21} & \alpha_{22} & * & * & * & * \\
0 & 0 & \beta & \gamma_{1} & \gamma_{2} & * \\
0 & 0 & 0 & \alpha_{11} & \alpha_{12} & * \\
0 & 0 & 0 & \alpha_{11} & \alpha_{12} & * \\
0 & 0 & 0 & 0 & 0 & \beta^{q}
\end{array}\right): \alpha_{i, j}, \beta, \gamma_{i} \in K\right\}
$$

(2) In contrast, the full quiver

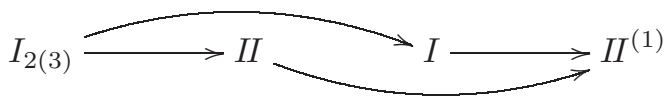

represents the algebra having all the relations of $A$, as well as the extra relation $\gamma_{1}=\gamma_{2}=0$, namely

$$
\left\{\left(\begin{array}{cccccc}
\alpha_{11} & \alpha_{12} & * & * & * & * \\
\alpha_{21} & \alpha_{22} & * & * & * & * \\
0 & 0 & \beta & 0 & 0 & * \\
0 & 0 & 0 & \alpha_{11} & \alpha_{12} & * \\
0 & 0 & 0 & \alpha_{11} & \alpha_{12} & * \\
0 & 0 & 0 & 0 & 0 & \beta^{q}
\end{array}\right): \alpha_{i, j}, \beta \in K\right\}
$$

(This is seen most easily by erasing the middle straight arrow in (3); now two of the curved arrows become primitive, so must be retained on notating the full quiver.) 
3.4. Proportional Frobenius gluing (of arrows). Like vertices, arrows correspond to matrix blocks defined over $K$, and are said to be of finite type if they can be defined over a finite subfield of $K$; otherwise they are said to be of infinite type. As with vertices, the complications in gluing arise for arrows of finite type.

Proposition 3.11. If $\Gamma$ is the full quiver of a representation of an algebra $A$, and if two primitive arrows in $\Gamma$ are proportionally Frobenius glued with respect to $q^{\ell}, q^{\ell^{\prime}}$ in Definition 3.3(6), then their respective vertices are glued with the same Frobenius twist.

Proof. Suppose that for some constant $\nu \in K$ we have $b_{r^{\prime}, s^{\prime}}=\nu b_{r^{\prime \prime}, s^{\prime \prime}}$ for every $a=\sum_{r, s} b_{r, s} \in A$, where $b_{r, s} \in B_{r, s}$. Since the diagonal blocks of the representation each are simple algebras, we may assume that $b_{r, r} \in F_{r}$. If the algebra $A$ contains an element $\hat{a}$ whose diagonal component is $\sum \hat{b}_{r} e_{r}$, again assuming that $\hat{b}_{r} \in F_{r}$, we can normalize to assume $b_{r^{\prime}, r^{\prime}}=\hat{b}_{r^{\prime}}$. But $A$ also contains $\hat{a} a=\sum_{r, s} \hat{b}_{r} b_{r, s}$, so taking $\hat{a} a-a^{2}$, we note that the $\left(r^{\prime}, s^{\prime}\right)$ coefficient is 0 whereas the $\left(r^{\prime \prime}, s^{\prime \prime}\right)$ coefficient is nonzero, and we have "unglued" the $r^{\prime \prime}, s^{\prime \prime}$ block from the $r^{\prime}, s^{\prime}$ block unless $\hat{b}_{r^{\prime \prime}}=b_{r^{\prime}}$.

The same argument holds for the second entry, when we fix $r$ and let $s$ vary.

Remark 3.12. The same argument of "ungluing" yields somewhat more precise information. Namely, with notation as in Remark 2.16, it shows that the block corresponding to the primitive arrow must belong to a field extension $L$ of the field of order $q^{\operatorname{gcd}\left(t_{r}, t_{s}\right)}$; hence $|L|=q^{c \cdot \operatorname{gcd}\left(t_{r}, t_{s}\right)}$ for some $c$ or $L=K$ is infinite.

Furthermore, the Frobenius twist of the arrows must equal $q^{\left(\ell^{\prime}-\ell\right)+m \cdot \operatorname{gcd}\left(t_{r}, t_{s}\right)}$ for some $m$, since otherwise multiplying by a diagonal component would "unglue" the arrows.

3.5. Synopsis of the full quiver. To summarize, the full quiver of $A \subseteq \mathrm{M}_{n}(K)$ is a graph without cycles which displays the following information:

(1) A vertex for each of the $\ell$ diagonal blocks, recording diagonal gluing;

(2) For each glued component $\mathbf{i}$ : the size $n_{\mathbf{i}}$ of its blocks, and the order $q^{u_{\mathbf{i}}}$ of the underlying field (which is $K$ or a finite extension of $F$, when the latter is finite);

(3) The identifications arising from Frobenius gluing along the diagonal;

(4) An arrow (directed edge) $r \rightarrow s$ connecting the $r, s$ vertices, for $r<s$, iff the block $B_{r, s} \neq 0$. Only primitive arrows are notated, as well as glued imprimitive arrows.

(5) The identifications arising from proportional Frobenius gluing above the diagonal.

3.6. The full quiver covers the classical quiver. Let us see how the full quiver covers the classical quiver, while providing more combinatoric information about the algebra.

Remark 3.13. One can interpret the components of the full quiver in terms of the structure of the corresponding algebra. Primitive arrows correspond to components of $J \backslash J^{2}$, in analogy to the classical quiver.

Remark 3.14. For any algebra $A$ over an infinite field $F$, the classical quiver of $A$ is the homomorphic image (as a graph) of the full quiver of any faithful representation 
of $A$. (When the base field $F$ is finite, this assertion may fail for two reasons: We may have nonidentical Frobenius gluing, and the classical quiver need not be finite, because of infinite dimensionality of the center of a block over $F$; see Example 5.4.)

The main difference from classical quivers, and the reason that we use full quivers of representations, is that by opening up glued components, each full quiver is a directed tree (i.e., has no directed cycle), and has no multiple arrows. This is clear from the definition and will be illustrated in our examples.

Example 3.15. The algebra

$$
A_{1}=\left\{\left(\begin{array}{cccc}
\alpha & * & * & * \\
0 & \beta & * & * \\
0 & 0 & \alpha & * \\
0 & 0 & 0 & \beta^{q}
\end{array}\right): \alpha \in \mathbb{F}_{q^{3}}, \beta \in K\right\}
$$

taken over $F=\mathbb{F}_{q}$ (where $K=\bar{F}$ ), which is Morita equivalent to Example 3.10(1), has full quiver notated as

$$
I_{(3)} \longrightarrow I I \longrightarrow I \longrightarrow I I^{(1)} .
$$

If instead we took

$$
K A_{1}=\left\{\left(\begin{array}{cccc}
\alpha & * & * & * \\
0 & \beta & * & * \\
0 & 0 & \alpha & * \\
0 & 0 & 0 & \beta^{q}
\end{array}\right): \alpha, \beta \in K\right\},
$$

as an algebra over $K$, then our full quiver would be notated as

$$
I \longrightarrow I I \longrightarrow I \longrightarrow I I^{(1)} .
$$

The classical quiver for this algebra is

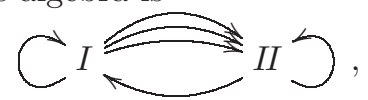

which we get by making the appropriate identifications in the full quiver (without first suppressing imprimitive arrows).

\section{Example 3.16.}

$$
A=\left\{\left(\begin{array}{cccc}
\alpha & * & * & * \\
0 & \beta & 0 & * \\
0 & 0 & \beta & * \\
0 & 0 & 0 & \alpha
\end{array}\right): \alpha, \beta \in K\right\},
$$

whose full quiver is notated as

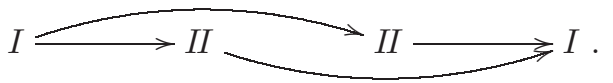

The classical quiver for this algebra is

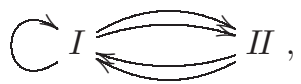

which again we get by making the appropriate identifications in the full quiver (without first suppressing imprimitive arrows). 


\subsection{Sub-quivers.}

Definition 3.17. A morphism $\psi: \Gamma_{1} \rightarrow \Gamma_{2}$ of full quivers is a morphism of directed graphs (i.e., if $\mathbf{i} \rightarrow \mathbf{j}$ is an arrow, then $\psi(\mathbf{i}) \rightarrow \psi(\mathbf{j})$ is also an arrow), preserving gluing, such that if $n_{\mathbf{i}}\left(t_{\mathbf{i}}\right)$ is the subscript for the vertex $\mathbf{i}$, then $\mathrm{M}_{n_{i}}\left(\mathbb{F}_{q^{t_{i}}}\right)$ embeds into $\mathrm{M}_{n_{\psi(\mathbf{i})}}\left(\mathbb{F}_{q^{t} \psi(\mathbf{i})}\right)$, whereby the superscripts are carried with their respective vertices.

We say that $\Gamma_{1}$ is a sub-quiver of $\Gamma_{2}$ if there is a 1:1 morphism from $\Gamma_{1}$ to $\Gamma_{2}$.

The infinite sub-quiver of a full quiver is the sub-quiver comprised of infinite vertices (i.e., vertices $e_{r}$ for which the corresponding field $F_{r}$ is infinite) together with the arrows between them.

Note that a morphism could send a primitive arrow to an imprimitive arrow.

Example 3.18.

(1) $I_{3(2)} \rightarrow I I_{(5)} \rightarrow I I I_{(3)}$ is a sub-quiver of

$$
I \rightarrow I I_{4(4)} \rightarrow I I I_{(5)} \rightarrow I V \rightarrow V_{(6)},
$$

under the morphism sending $I \mapsto I I, I I \mapsto I I I$, and $I I I \mapsto V$, but is not a sub-quiver of $I \rightarrow I_{4(4)} \rightarrow I I I_{(5)} \rightarrow I V \rightarrow V_{(2)}$.

(2) $\bullet_{(4)}$ is a sub-quiver of $\bullet_{2(2)}$ because of the regular representation $\mathbb{F}_{q^{4}} \subseteq$ $\mathrm{M}_{2}\left(\mathbb{F}_{q^{2}}\right)$.

\subsection{Full quivers viewed structurally.}

Remark 3.19. Note that the quivers come from components of the affine variety defined by the Zariski closed algebra, which can be described in terms of Remark 2.18. The vertices come from the semisimple components, which are just matrix algebras. However, the arrows are somewhat more complicated.

In principle, the arrows correspond to matrix units arising in the representation corresponding to the given Wedderburn block form. When the base field $F$ is infinite, then $K=F$, and the Zariski closure is just a finite dimensional algebra; in this case, any arrow can be identified with the appropriate element in the algebra. We call this the element of $A$ corresponding to the arrow.

But when $F$ is finite, the situation is subtler. We could have a situation such as

$$
A=\left\{\left(\begin{array}{cc}
F & K \\
0 & K
\end{array}\right)\right\} \subset \mathrm{M}_{2}(K)
$$

whose full quiver is

$$
\bullet_{(1)} \longrightarrow
$$

Note that when $F_{1} \subset F_{2} \subset K$ we get the same full quivers

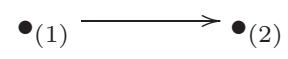

from the algebras $\left\{\left(\begin{array}{cc}F_{1} & F_{2} \\ 0 & F_{2}\end{array}\right)\right\}$ and $\left\{\left(\begin{array}{cc}F_{1} & K \\ 0 & F_{2}\end{array}\right)\right\}$, so we cannot recover the radical component from the arrow.

In this case, we view the arrows as corresponding to linear functionals, and we often are led to use the generic elements obtained in [9, Construction 7.14]. By definition, since any generic element is taken from a given component in the affine variety defined by the Zariski closure, the choice of generic element for this component does not affect the polynomial relations. Thus the gluing does not depend on the particular choice of generic element corresponding to a given arrow. 
Here is a more precise way of viewing the last paragraph of the previous remark.

Let us record a monoid action needed for subsequent papers.

Remark 3.20. Let $\mathcal{M}_{i}$ denote the multiplicative monoid $\mathbb{Z} / q^{t_{i}} \mathbb{Z} \cup\{0\}$. Then $\mathcal{M}_{k}$ acts naturally on $F_{k}$ via $[j] a=a^{j}$, where $a^{0}=1$. Consequently, $\mathcal{M}_{1} \times \cdots \times \mathcal{M}_{k}$ acts on $A$, leading to a grading in terms of the sub-Peirce decomposition and Frobenius gluing. In [11] we use this grading to study the polynomial identities of $A$.

\section{SPeCific Classes of FUll QUivers}

In this section, we describe various classes of full quivers, starting with the easiest case, and then increasing in complexity. A full quiver is called a path if its initial and terminal vertices each have degree 1 and all other vertices have degree 2 . Let us consider some examples of paths and other relatively simple examples of full quivers.

4.1. Full quivers of semisimple algebras. Having described representations of semisimple algebras in Remark 2.11 we can easily give their full quivers, which are just finite sets of disconnected vertices (since the radical $J=0$ ). The full quiver of a matrix algebra is a single bullet $\bullet$ without any arrows. The full quiver of a direct sum of matrix algebras is just a set of disconnected bullets, in view of Remark 3.6.

4.2. Elementary full quivers. After the case of unconnected vertices without arrows, the next most basic kind of full quiver, called elementary, is a full quiver that consists of a single arrow; this corresponds to module and bimodule structures over a semisimple algebra. Any algebra having an elementary full quiver satisfies $J^{2}=0$; let us consider the various possibilities.

(1) No gluing. The full quiver $I_{m\left(t_{1}\right)} \rightarrow I I_{n\left(t_{2}\right)}$ corresponds to the algebra

$$
A=\left(\begin{array}{cc}
\mathrm{M}_{m}\left(F_{1}\right) & L \\
0 & \mathrm{M}_{n}\left(F_{2}\right)
\end{array}\right),
$$

where the $F_{i}$ are intermediate subfields of $F \subseteq K$ of order $|F|^{t_{i}}$ (or are infinite in the absence of the $\left.t_{i}\right)$, and $L$ is a nonzero $\mathrm{M}_{m}\left(F_{1}\right), \mathrm{M}_{n}\left(F_{2}\right)$ subbimodule of the upper right part of $\mathrm{M}_{m+n}(K)$. The defining relations are $\lambda_{m+i^{\prime}, j}=0, \lambda_{i j}^{\left|F_{1}\right|}=\lambda_{i j}$, and $\lambda_{m+i^{\prime}, m+j^{\prime}}^{\left|F_{2}\right|}=\lambda_{m+i^{\prime}, m+j^{\prime}}$, for all $1 \leq i, j \leq$ $m, 1 \leq i^{\prime}, j^{\prime} \leq n$ (indeed, these relations say that the first diagonal block has coefficients in $F_{1}$ and the second diagonal block has coefficients in $F_{2}$ ), and the extra relations defining $L$.

(2) Identical gluing. The full quiver $I_{n(t)} \rightarrow I$ corresponds to the algebra $A$ as in (1), but with $m=n$, and with gluing. Note that $A \subseteq \mathrm{M}_{n}\left(K[\lambda] /\left\langle\lambda^{2}\right\rangle\right)$. (If $F$ is infinite, then $\operatorname{id}(A)=\operatorname{id}\left(\mathrm{M}_{n}(F)\right.$. If $\left|F_{1}\right|=q$, and $g$ is a central polynomial for $M_{n}\left(F_{1}\right)$, then $g^{q}-g \in \operatorname{id}\left(M_{n}\left(F_{1}\right)\right)$ but is not in $\operatorname{id}(A)$.)

For $m=n=1$ we have

$$
A=\left\{\left(\begin{array}{cc}
\alpha & \beta \\
0 & \alpha
\end{array}\right): \alpha \in F_{1}, \beta \in L\right\},
$$

where $F_{1}$ is as before, and $F_{1} \subseteq L \subseteq K$ is any (Zariski closed) intermediate field. We have the same relations in (1), plus $\lambda_{22}=\lambda_{11}$. 
(3) Frobenius gluing. The full quiver $I_{n_{1}\left(t_{1}\right)} \rightarrow I^{(u)}$ corresponds to the algebra analogous to (2), but with Frobenius gluing of degree $\ell$. For $m=n=1$ we have

$$
A=\left\{\left(\begin{array}{cc}
\alpha & \beta \\
0 & \alpha^{\pi}
\end{array}\right): \alpha \in F_{1}, \beta \in L\right\}
$$

where $F_{1}, L$ are as before, and $\pi=q^{u}$. We have the same relations as for (2), with $\lambda_{22}=\lambda_{11}^{\pi}$ replacing $\lambda_{22}=\lambda_{11}$. Note that

$$
\left(\begin{array}{cc}
\alpha & \beta \\
0 & \alpha^{\pi}
\end{array}\right)\left(\begin{array}{cc}
\alpha^{\prime} & \beta^{\prime} \\
0 & \alpha^{\prime \pi}
\end{array}\right)=\left(\begin{array}{cc}
\alpha \alpha^{\prime} & \alpha \beta^{\prime}+\beta \alpha^{\prime \pi} \\
0 & \alpha^{\pi} \alpha^{\prime \pi}
\end{array}\right),
$$

so in some sense the multiplication on the $(1,2)$ component is "skewed" by a $\pi$-power. For general $m$ and $n$ we take $\pi$-powers of the corresponding coordinates.

(4) $\circ \rightarrow \bullet$ corresponds to the algebra $\left\{\left(\begin{array}{ll}0 & * \\ 0 & *\end{array}\right)\right\}$ without 1 . Similarly, $\bullet \rightarrow \circ$ corresponds to the algebra $\left\{\left(\begin{array}{ll}* & * \\ 0 & 0\end{array}\right)\right\}$ without 1 . (These are similar to (11), where one of the diagonal components is 0 . Here, $\operatorname{id}(A)$ is $x \operatorname{id}\left(M_{n}\left(F_{1}\right)\right)$ and $\operatorname{id}\left(M_{n}\left(F_{1}\right)\right) x$, respectively, by Lewin's Theorem.)

$(5) \circ \rightarrow \circ$ corresponds to the set of matrices whose only nonzero entry is in the upper right corner of $2 \times 2$ matrices. (Here, $\mathrm{id}(A)=x y$.)

4.3. Branches of full quivers. In order to investigate full quivers further, we need the following important notion.

Definition 4.1. A path in a full quiver is a branch if it cannot be extended, namely if no arrow enters its first vertex, and no arrow exits its final vertex. The length of a path is its number of arrows, which is one less than the number of vertices. (Thus a branch of length $\ell-1$ has $\ell$ vertices.) Likewise, an arrow has length $\ell$ if there is a path from $i$ to $j$ having length $\ell$.

Remark 4.2. Since radical elements correspond to arrows, we see that the index of nilpotence of an algebra is at most $\ell+1$, where $\ell$ is the maximal length of the paths of its full quiver.

Remark 4.3. Given a single branch with Frobenius proportional gluing, one can make all the parameters equal to 1 via a change of base. Thus, in this case, pure proportional gluing in any given branch becomes identical gluing.

\section{Gluing, COMpression of Full Quivers, And Change of Base Ring}

The definition of quivers is combinatoric. Although we have defined full quivers of linear representations over a field, the same definition applies to linear representations over any commutative affine algebra, such that all entries are given in blocks along or above the diagonal.

Closely related representable algebras (involving gluing) could require (faithful) representations of widely varying degrees, which we can describe better in terms of representations of much lower degree over arbitrary commutative affine algebras. Let us first describe commutative affine algebras in terms of full quivers of representations. We start with unglued arrows. 
Proposition 5.1. An indecomposable algebra (see Remark 3.6) with unglued arrows is commutative iff all of its vertices are identically glued and every branch has length $\leq 1$

Proof. $(\Rightarrow)$ If we have an arrow between unglued vertices, then the algebra cannot be commutative, since $e_{i i} e_{i j}=e_{i j}$ whereas $e_{i j} e_{i i}=0$. In light of Remark 3.6. this means all vertices must be glued; the gluing must all be identical, because $\alpha e_{i i}+\alpha^{s} e_{j j}$ does not commute with $e_{i j}$ for $\alpha^{s} \neq \alpha$. Moreover, the same argument $\left(e_{i j} e_{j k}=e_{i k}\right.$ whereas $\left.e_{j k} e_{i j}=0\right)$ shows that there cannot be two consecutive arrows.

$(\Leftarrow)$ is obvious.

Example 5.2. The full quivers of Proposition 5.1 are bi-partite, with disjoint sets of sources and sinks. For example,

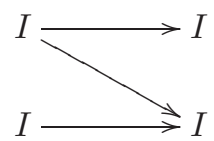

or

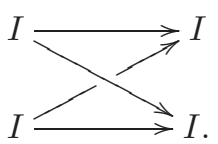

5.1. Compressed full quivers. When glued arrows are allowed, there are many other possibilities for representations of commutative algebras.

Example 5.3. The local commutative algebra $F[\lambda] /\left\langle\lambda^{4}\right\rangle$ has the presentation

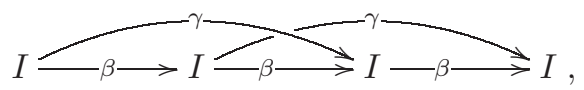

in which the $(1,2),(2,3)$ and $(3,4)$ entries are identically glued, as well as the $(1,3)$ and $(2,4)$ entries. (The nonprimitive arrow of length 2 is omitted from the diagram.) Omitting all the nonprimitive arrows, as in

$$
I \longrightarrow \beta \longrightarrow I \longrightarrow \beta \longrightarrow I \longrightarrow \beta \longrightarrow I \text {, } \longrightarrow \text {, }
$$

would release the gluing of length-1 arrows, yielding a 4-dimensional algebra which is not commutative. Frobenius gluing of the length-1 arrows also yields noncommutative algebras.

Example 5.4. Generalizing the previous example, gluing within a branch leads to the following sort of situation:

$$
B_{\ell}=\left\{\left(\begin{array}{ccccc}
\alpha & \beta & \gamma & \ldots & \cdots \\
0 & \alpha & \beta & \ddots & \vdots \\
0 & 0 & \ddots & \ddots & \gamma \\
0 & 0 & 0 & \alpha & \beta \\
0 & 0 & 0 & 0 & \alpha
\end{array}\right): \alpha, \beta, \gamma \in K\right\} .
$$

This algebra is isomorphic to $F[\lambda] /\left\langle\lambda^{\ell}\right\rangle$, where $\ell$ is the dimension of the matrices. We call the full quiver of this algebra the glued triangle $I(\ell)$; in the previous notation it is $I \rightarrow I \rightarrow \cdots \rightarrow I$, where all arrows of equal length are identically 
glued. We call the glued triangle $I(\ell)$ isolated if the vertex $I$ does not appear elsewhere in the branch. (Note for $|F|<\infty$ that $\operatorname{id}\left(B_{\ell}\right) \supset \operatorname{id}(F)$.)

(Allowing infinite dimensional representations, one can embed $F[\lambda]$ in $\operatorname{End}\left(F^{\omega}\right)$ by letting $\lambda$ be the shift forward operator, corresponding to the infinite full quiver $I(\infty)=I \rightarrow I \rightarrow \cdots$.)

Conversely, given an isolated glued triangle (with identical gluing) in a branch, we could compress it to a point (and erase any duplication of arrows) and also erase all imprimitive arrows, relabelling the vertex $\mathbf{i}_{d_{\mathbf{i}}\left(n_{\mathbf{i}}\right)}$ as $\mathbf{i}_{d_{\mathbf{i}}\left(n_{\mathbf{i}}\right)}(\ell)$, to indicate that, in that block, we have replaced the field $\mathbb{F}_{q_{\mathrm{i}}}$ by the commutative affine algebra $\mathbb{F}_{q_{\mathrm{i}}}[\lambda] /\left\langle\lambda^{\ell}\right\rangle=\mathbb{F}_{q_{\mathrm{i}}}\left[\varepsilon_{\ell}\right]$, where $q_{\mathbf{i}}=q^{n_{\mathbf{i}}}$ and $\left(\varepsilon_{\ell}\right)^{\ell}=0$. The resulting full quiver (after this process) is called the compressed quiver.

Example 5.5. Compression of the glued triangle eliminates redundant, identically glued, imprimitive arrows originating or ending in the compressed idempotents. Therefore, one can compress

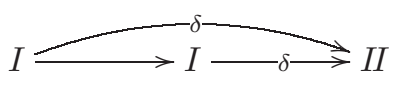

to

$$
I(2) \stackrel{\delta}{\longrightarrow} I I
$$

and

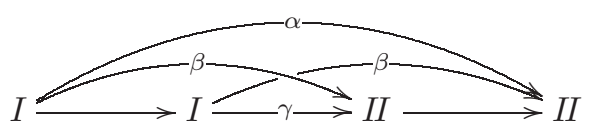

to

$$
I(2) \longrightarrow I I(2)
$$

but

$$
I \longrightarrow I \longrightarrow I I
$$

which is a shorthand notation for

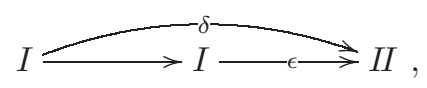

cannot be compressed.

Note that any morphism of full quivers naturally induces a morphism of compressed quivers.

Remark 5.6. As noted in Example 5.4. compression can enable us to decrease the degree of a representation, at the expense of introducing various commutative affine algebras as base rings for the compressed vertices.

Compression of a branch involves adjoining elements $\varepsilon_{i, \ell}$ to the base field $F$, for various $\ell$, such that $\varepsilon_{i, \ell}^{\ell}=0$. Thinking of these $\varepsilon_{i, \ell}$ as "infinitesimals of order $\ell$ ", we see that the compressed branch merely extends the base ring by adjoining various infinitesimals. The downside of this approach is that the algebraic interpretation of our full quivers becomes much more complicated, since part of the radical now belongs to the commutative base ring, and arrows no longer need correspond to 1:1 maps. 
Let us describe compression systematically, extracting the full potential of identical gluing. A local component of a given vertex $I$ is a maximal path of the form $I(\ell)$ in which it participates (a vertex can belong to more than one local component, as seen in Example 5.2). Let $P$ denote the set of vertices glued to a given vertex $I$. If:

- the local components of the vertices $\mathbf{i} \in P$ are disjoint;

- all the local components have the same length;

- arrows of the same length within all local components of the same length are identically glued;

- if $\mathbf{i}, \mathbf{i}^{\prime} \in P$ are in the same local component, and $\mathbf{j}, \mathbf{j}^{\prime} \in P$ are in the same local component, then the arrow from $\mathbf{i}$ to $\mathbf{j}$ is identically glued to the arrow from $\mathbf{i}^{\prime}$ to $\mathbf{j}^{\prime}$;

- for every $\mathbf{j} \notin P$, all arrows from $\mathbf{i} \in P$ to $\mathbf{j}$ (when present) are identically glued, and all arrows from $\mathbf{j}$ to $\mathbf{i} \in P$ (when present) are identically glued,

then we may compress all local components, labelling each by notation of the form $I(\ell)$. This process can be iterated, labelling a sub-quiver of the form $I(\ell) \rightarrow I(\ell)$ as $I(\ell)(2)$, etc., until no further compression is possible; then the full quiver is in incompressible form. The name 'local component' remains appropriate, since the algebra $R\left[\epsilon \mid \epsilon^{\ell}=0\right]$ is local whenever $R$ is local. Since the full quiver is finite, we have

Proposition 5.7. Every full quiver has a unique incompressible form.

Example 5.8. (1) The full quivers
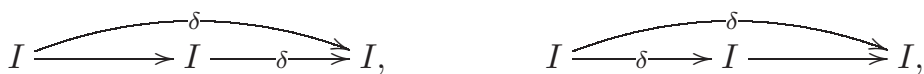

can be compressed to

$$
I(2) \longrightarrow I, \quad I \longrightarrow I(2),
$$

respectively.

(2) The full quiver

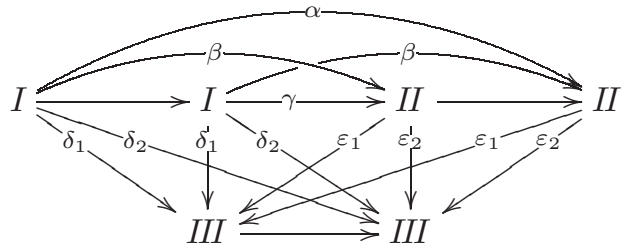

can be compressed to

$$
I(2) \longrightarrow I I(2) \longrightarrow I I(2),
$$

with the quadruple arrow denoted by $\alpha$ becoming imprimitive.

(3) Similarly,

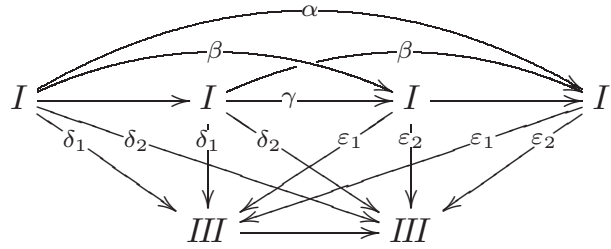


can be compressed to

$$
I(2) \longrightarrow \text { - } \mathrm{C}(2) \longrightarrow I I I(2),
$$

and further compressed to $I(2)(2) \rightarrow I I I(2)$.

5.2. Partial gluing up to infinitesimals. Consider a fixed vertex $I$ in a full quiver, and let $A_{0}$ be the subalgebra generated by all the sub-quivers of the form $I(\ell)$. The matrix algebra represented by $I$ (which may itself be an algebra over a local ring) is a homomorphic image of $A_{0}$, and the kernel (generated by the arrows) is nilpotent. When there is enough gluing so that this kernel is generated by one arrow, we say that all the components of $A_{0}$ are glued up to infinitesimals.

Example 5.9. Consider the following (incompressible) full quivers:

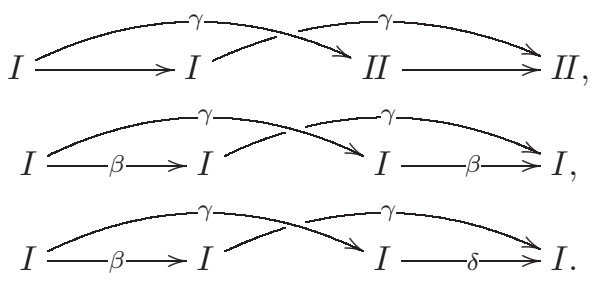

One can view the algebra corresponding to diagram (11) as

$$
\left\{\left(\begin{array}{cccc}
\alpha & \delta & \gamma & \nu \\
0 & \alpha & 0 & \gamma \\
0 & 0 & \beta & \mu \\
0 & 0 & 0 & \beta
\end{array}\right): \alpha, \beta, \gamma, \delta, \mu, \nu \in K\right\} .
$$

Partitioning these terms into $2 \times 2$ matrices enables us to rewrite this algebra as

$$
\left\{\left(\begin{array}{cc}
\phi & \chi \\
0 & \psi
\end{array}\right): \phi, \psi, \chi \in F\left[\varepsilon_{2}\right]\right\}
$$

where $\varepsilon_{2}$ satisfies the relation $\varepsilon_{2}^{2}=0$.

Likewise the algebra corresponding to diagram (12) can be viewed as

$$
\left\{\left(\begin{array}{llll}
\alpha & \delta & \gamma & \nu \\
0 & \alpha & 0 & \gamma \\
0 & 0 & \alpha & \delta \\
0 & 0 & 0 & \alpha
\end{array}\right): \alpha, \beta, \gamma, \delta, \nu \in K\right\},
$$

which can be rewritten as

$$
\left\{\left(\begin{array}{cc}
\phi & \chi \\
0 & \phi
\end{array}\right): \phi, \chi \in F\left[\varepsilon_{2}\right]\right\} .
$$

The algebra corresponding to diagram (13),

$$
\left\{\left(\begin{array}{cccc}
\alpha & \beta & \gamma & \nu \\
0 & \alpha & 0 & \gamma \\
0 & 0 & \alpha & \delta \\
0 & 0 & 0 & \alpha
\end{array}\right): \alpha, \beta, \gamma, \delta, \nu \in K\right\},
$$

can be presented with an extra relation, as

$$
\left\{\left(\begin{array}{cc}
\phi & \chi \\
0 & \psi
\end{array}\right): \phi, \psi, \chi \in F\left[\varepsilon_{2}\right],(\phi-\psi)^{2}=0\right\} \text {. }
$$


5.2.1. Generic elements used with partial gluing. In [9, Construction 7.19] we exhibit, for a Zariski closed algebra $A$ over a field $F$, an explicit generic algebra, generated by glued sums of sufficiently long products of generic elements. This algebra then generates the variety of $F$-identities of $A$. The easiest example is given below as (10) in Example 5.10, where we adjust the construction to more general base rings. When representing generic algebras, one may refine the gluing procedure, as indicated in the following sequence of examples.

Example 5.10. (1) The generic upper triangular $2 \times 2$ matrix $Y_{i}=\left(\begin{array}{cc}\xi_{i 1} & \xi_{i 3} \\ 0 & \xi_{i 2}\end{array}\right)$ is defined over the polynomial algebra $C=F\left[\xi_{i j}: i \in \mathbb{N}, 1 \leq j \leq 3\right]$; we get the generic algebra of upper triangular matrices by taking the subalgebra of $\mathrm{M}_{2}(C)$ generated by the $Y_{i}$.

(2) In (1), we can glue the two diagonal components, by replacing $C$ by the algebra

$$
\bar{C}=F\left[\xi_{i j}: i \in \mathbb{N}, 1 \leq j \leq 3\right] /\left\langle\xi_{i 1}-\xi_{i 2}\right\rangle .
$$

(3) We can modify the gluing procedure to leave only an "infinitesimal part" unglued. For example, let us replace $C$ by

$$
\bar{C}=F\left[\xi_{i j}: i \in \mathbb{N}, 1 \leq j \leq 3\right] /\left\langle\left(\xi_{i_{1}, 1}-\xi_{i_{1}, 2}\right)\left(\xi_{i_{2}, 1}-\xi_{i_{2}, 2}\right)\right\rangle .
$$

Setting $\xi_{i 2}=\xi_{1 i}+\varepsilon_{i}$, one could think of $\bar{C}$ as generated by $\xi_{i 1}, \xi_{i 3}$ and $\varepsilon_{i}$ $(i \in \mathbb{N})$, where $\varepsilon_{i_{1}} \varepsilon_{i_{2}}=0$ for all $i_{1}, i_{2}$, and then the algebra of matrices is generated by $Y_{i}=\left(\begin{array}{cc}\xi_{i 1} & \xi_{i 3} \\ 0 & \xi_{i 1}+\varepsilon_{i}\end{array}\right)$. Thus we view the $\varepsilon_{i}$ as "infinitesimal elements of second order", in the sense that the product of any two of them is zero. From this point of view, we are gluing the two diagonal entries up to infinitesimals.

This example is symmetric, in the sense that the matrices

$$
Y_{i}=\left(\begin{array}{cc}
\xi_{i 2}-\varepsilon_{i} & 0 \\
0 & \xi_{i 2}
\end{array}\right)
$$

so one could reverse the roles of $\xi_{i 1}$ and $\xi_{i 2}$.

(4) More generally, for any $k>1$, take

$$
\bar{C}=F\left[\xi_{i 1}, \xi_{i 2}, \xi_{i 3}: i \in I\right] /\left\langle\left(\xi_{i 1}-\xi_{i 2}\right): i \in I\right\rangle^{k} .
$$

One could think of the algebra of (3) instead as generated by matrices $Y_{i}=\left(\begin{array}{cc}\xi_{i 1} & \xi_{i 3} \\ 0 & \xi_{i 1}+\varepsilon_{i}\end{array}\right)$, where $\left(\sum F \varepsilon_{i}\right)^{k}=0$. Thus, we view the $\varepsilon_{i}$ as "infinitesimal elements of order $k$ ", in the sense that the product of any $k$ of them is zero.

(5) Consider the ideal $I$ of the commutative polynomial algebra $F\left[\xi_{i j}^{(\ell)}\right]$, generated by the $\xi_{i j}^{(\ell)}{ }^{q}-\xi_{i j}^{(\ell)}$. The algebra $F\left[\xi_{i j}^{(\ell)}\right] / I^{k}$ can be viewed as obtained by deforming a finite field by "infinitesimal elements of order $k$ ".

(6) We can also consider generic upper triangular matrices with gluing of the diagonal entries up to infinitesimals, by adjoining extra indeterminates to $\bar{C}$ and considering matrices of the form

$$
\left(\begin{array}{cc}
\xi_{i 1} & \xi_{i 3} \\
0 & \xi_{i 1}+\epsilon
\end{array}\right)
$$


represented over a field, these could be viewed as $3 \times 3$ matrices of the form

$$
\left(\begin{array}{ccc}
\xi_{i 1} & \xi_{i 3} & \xi_{i 3} \\
0 & \xi_{i 1} & \xi_{i 2} \\
0 & 0 & \xi_{i 1}
\end{array}\right)
$$

(7) We can complicate (11), taking instead the algebra generated by matrices

$$
Y_{i}=\left(\begin{array}{ccc}
\xi_{i 1} & * & * \\
0 & \xi_{i 2} & * \\
0 & 0 & \xi_{i 2}+\varepsilon_{i}
\end{array}\right)
$$

where the $\varepsilon_{i}$ are "infinitesimal elements of second order", and * denotes arbitrary entries.

The algebra of Example 5.1000 in represented in $\mathrm{M}_{3}\left(F\left[\varepsilon_{i}\right]\right)$. Since $\varepsilon_{i}$ is nilpotent of order $2, \varepsilon_{i}$ can be identified in $\mathrm{M}_{2}\left(F\left(\xi_{i}\right)\right.$ ) (for $\xi_{i}$ a commuting indeterminate over $F)$ with $\left(\begin{array}{ll}0 & \xi \\ 0 & 0\end{array}\right)$. Thus, Example 5.10,7) has a faithful representation of degree 6 over a field. (In fact, one could get a representation of degree 4 using the argument of Example 5.1066).) When we increase the order of an infinitesimal, we must increase the order of the faithful representation (over a field) accordingly, since an infinitesimal $\varepsilon$ of order $k$ is represented by the $k \times k$ matrix

$$
\left(\begin{array}{ccccc}
0 & \xi & 0 & \ldots & 0 \\
0 & 0 & \xi & \ldots & 0 \\
\vdots & \vdots & \vdots & \ddots & \vdots \\
0 & 0 & 0 & \ldots & \xi \\
0 & 0 & 0 & \ldots & 0
\end{array}\right)
$$

which is nilpotent of order $k$. (Note that as the order of the representation increases, so does the index of nilpotence of the radical.)

The generic description of partial gluing up to infinitesimals becomes more complicated in the presence of a slight generalization of Frobenius gluing, because we need to deal both with the Frobenius automorphism and also with the degree of the infinitesimal.

Example 5.11. In order to introduce Frobenius gluing of power $q$, up to infinitesimals, to Example 5.10] (6), we define generic triangular matrices $Y_{i}=\left(\begin{array}{cc}\xi_{i 1} & \xi_{i 3} \\ 0 & \xi_{i 1}^{q}+\varepsilon_{i}\end{array}\right)$, where each $\varepsilon_{i} \varepsilon_{j}=0$; thus, we replace $C$ by the algebra

$$
\bar{C}=F\left[\xi_{i j}: i \in I, 1 \leq j \leq 3\right] /\left\langle\xi_{i_{1}, 1}^{q}-\xi_{i_{1}, 3}\right\rangle^{2} .
$$

Stipulating the base field $F$ to have order $q$ requires extra relations of the form $\left(\xi_{i j}^{q}-\right.$ $\left.\xi_{i j}\right)$.

To describe the representation over a field, we must pass to

$$
\left\{\left(\begin{array}{ccc}
\alpha & * & * \\
0 & \alpha^{q} & * \\
0 & 0 & \alpha^{q}
\end{array}\right): \alpha \in F\right\} .
$$

The full quiver for this representation is the path

$$
I \longrightarrow I^{(1)} \longrightarrow I^{(1)} \text {. }
$$


One can also introduce Frobenius "self-gluing" of power $q$, up to infinitesimals, by imposing the relation $\left(x^{q}-x\right)^{\ell}$ on a vertex.

All examples of infinitesimal compression can be viewed in such a manner, where the number of identifications at the end is the order of the infinitesimal.

5.3. Proportional gluing. Off-diagonal gluing involves extra intricacies. Here is an example of a commutative algebra with nonidentical gluing.

Example 5.12. Fix $\nu \in K$. The algebra

$$
\left\{\left(\begin{array}{ccc}
\alpha & \beta & \gamma \\
0 & \alpha & \nu \beta \\
0 & 0 & \alpha
\end{array}\right): \alpha, \beta, \gamma \in K\right\}
$$

is a commutative algebra which is described by the full quiver

$$
I \stackrel{\beta}{\longrightarrow} I \stackrel{\nu \beta}{\longrightarrow} I,
$$

which denotes the relation $\lambda_{21}=\nu \lambda_{32}$. Indeed, to verify commutativity, one may assume $\alpha=0$ and check that

$$
\left(\begin{array}{ccc}
0 & \beta & \gamma \\
0 & 0 & \nu \beta \\
0 & 0 & 0
\end{array}\right)\left(\begin{array}{ccc}
0 & \beta^{\prime} & \gamma^{\prime} \\
0 & 0 & \nu \beta^{\prime} \\
0 & 0 & 0
\end{array}\right)=\left(\begin{array}{ccc}
0 & 0 & \nu \beta \beta^{\prime} \\
0 & 0 & 0 \\
0 & 0 & 0
\end{array}\right)=\left(\begin{array}{ccc}
0 & \beta^{\prime} & \gamma^{\prime} \\
0 & 0 & \nu \beta^{\prime} \\
0 & 0 & 0
\end{array}\right)\left(\begin{array}{ccc}
0 & \beta & \gamma \\
0 & 0 & \nu \beta \\
0 & 0 & 0
\end{array}\right) .
$$

Example 5.13. We start with the paths

and

$$
I \longrightarrow I \longrightarrow I \longrightarrow \text {. }
$$

We recall that the full quiver of the diagram (17) corresponds to the algebra of upper triangular matrices; the full quiver of the diagram (18), in which the blocks are glued, corresponds to the algebra of unipotent upper triangular matrices

$$
U_{4}=\left\{\left(\begin{array}{cccc}
\alpha & * & * & * \\
0 & \alpha & * & * \\
0 & 0 & \alpha & * \\
0 & 0 & 0 & \alpha
\end{array}\right): \alpha \in F\right\}
$$

without radical gluing.

Identical labels in the full quiver (18) provide identifications in the next layer of components, such as for the algebra

$$
U_{4}^{+}=\left\{\left(\begin{array}{cccc}
\alpha & \beta & \gamma & \mu \\
0 & \alpha & \beta & \delta \\
0 & 0 & \alpha & \beta \\
0 & 0 & 0 & \alpha
\end{array}\right): \alpha, \beta, \gamma, \delta, \mu \in F\right\}
$$

whose full quiver is given in (10).

In order to add the relation $\delta=\gamma$, we must bring the nonprimitive arrows back into the full quiver, which no longer can be represented via a single branch, as illustrated in diagram (9). 
But this can be compressed to a point. The corresponding algebra

$$
B_{4}=\left\{\left(\begin{array}{cccc}
\alpha & \beta & \gamma & \mu \\
0 & \alpha & \beta & \gamma \\
0 & 0 & \alpha & \beta \\
0 & 0 & 0 & \alpha
\end{array}\right): \alpha, \beta, \gamma, \mu \in F\right\}
$$

is commutative, being isomorphic to the algebra $F[\varepsilon] /\left\langle\varepsilon^{4}\right\rangle$.

If instead we want the relation $\delta=\nu \gamma$, we get the full quiver:

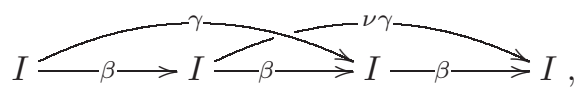

whose corresponding algebra is still commutative, since

$$
\begin{aligned}
\left(\begin{array}{cccc}
0 & \beta & \gamma & \mu \\
0 & 0 & \beta & \nu \gamma \\
0 & 0 & 0 & \beta \\
0 & 0 & 0 & 0
\end{array}\right) & \left(\begin{array}{cccc}
0 & \beta^{\prime} & \gamma^{\prime} & \nu \\
0 & 0 & \beta^{\prime} & \nu \gamma^{\prime} \\
0 & 0 & 0 & \beta^{\prime} \\
0 & 0 & 0 & 0
\end{array}\right)=\left(\begin{array}{cccc}
0 & 0 & \beta \beta^{\prime} & \nu\left(\beta \gamma^{\prime}+\beta^{\prime} \gamma\right) \\
0 & 0 & 0 & \beta \beta^{\prime} \\
0 & 0 & 0 & 0 \\
0 & 0 & 0 & 0
\end{array}\right) \\
& =\left(\begin{array}{cccc}
0 & \beta^{\prime} & \gamma^{\prime} & \nu \\
0 & 0 & \beta^{\prime} & \nu \gamma^{\prime} \\
0 & 0 & 0 & \beta^{\prime} \\
0 & 0 & 0 & 0
\end{array}\right)\left(\begin{array}{cccc}
0 & \beta & \gamma & \mu \\
0 & 0 & \beta & \nu \gamma \\
0 & 0 & 0 & \beta \\
0 & 0 & 0 & 0
\end{array}\right)
\end{aligned}
$$

5.4. Gluing between branches - Permuted gluing. The theory becomes considerably more intricate when we need to consider gluing between several different branches in the same full quiver. The same kinds of gluing (identical, Frobenius, proportional Frobenius) are involved.

Definition 5.14. Gluing between branches is called total if for each arrow in one branch there is an arrow of the other branch, glued proportionally to it.

Total gluing need not involve a 1:1 correspondence of the arrows, e.g., the algebra

$$
\left\{\left(\begin{array}{ccccc}
\alpha & \beta & * & \gamma & * \\
0 & \alpha & \beta & 0 & * \\
0 & 0 & \alpha & 0 & \gamma \\
0 & 0 & 0 & \alpha & \beta \\
0 & 0 & 0 & 0 & \alpha
\end{array}\right): \alpha, \beta, \gamma \in F\right\}
$$

whose full quiver is

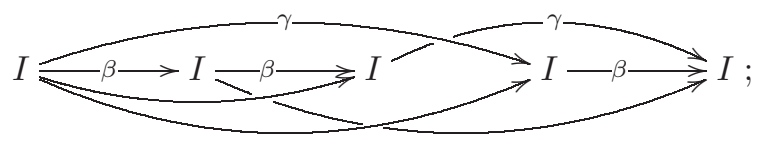

as usual, undecorated arrows stand for nonglued entries.

Definition 5.15. Total gluing between two branches is permuted if it involves a permutation of the arrows. (In other words, there is a graph isomorphism which preserves the labels of vertices, and which also preserves the labels of arrows up to multiplying by constants.)

Degenerate gluing is the special case of permuted gluing in which one branch is glued identically, arrow for arrow, with another branch (i.e., via the identity permutation). 
Degenerate gluing is illustrated in the following quiver, for fixed $\lambda, \lambda^{\prime} \in F$ :

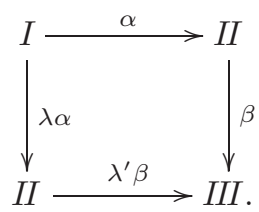

Lemma 5.16. Any full quiver is a union of full quivers with total gluing.

Proof. We say that two branches are equivalent if they have permuted gluing between them. This is obviously an equivalence, so we just partition the branches of the full quiver by their equivalence classes.

When dealing with degenerate gluing of paths, there are two separate cases to consider.

Example 5.17. Consider the matrix representation of the full quiver (21), where the components form the diagonal blocks of $\mathrm{M}_{4}(K)$ in the natural manner. According to the given proportional gluing, the radical is generated by $e_{12}+\lambda e_{13}$ and $e_{24}+\lambda^{\prime} e_{34}$, whose product is $\left(1+\lambda \lambda^{\prime}\right) e_{14}$. If $1+\lambda \lambda^{\prime} \neq 0$, then the algebra is isomorphic to the one represented via the full quiver $I \rightarrow I I \rightarrow I I I$. Otherwise, there is no map from $I$ to $I I I$, and the algebra can be represented via the glue-connected full quiver $I \rightarrow I I, I I \rightarrow I I I$.

Using generic commuting elements to expand the base ring, one can remove all degenerate gluing in a more systematic manner.

Remark 5.18. One can transform the degenerate gluing of paths in a full quiver into a single path (at the expense of passing to algebras over commutative affine algebras which are not necessarily fields). Namely: if the products of the primitive arrows sum to a nonzero map, replace the glued paths by a single branch having the same attributions. Otherwise, if the sum is zero, attach a formal commuting indeterminate $\xi_{i}$ to each gluing class of arrows, and define the appropriate relations on the commutative polynomial algebra $F[\xi]$ to obtain a commutative affine algebra for which the degeneration is described in terms of a single branch.

For example, for the full quiver given in (21) we replace $\alpha$ by $\xi_{1} \alpha$ and $\beta$ by $\xi_{2} \beta$, and let $C=F\left[\xi_{1}, \xi_{2}\right] /\left\langle\xi_{1} \xi_{2}\right\rangle$. Then we can represent our algebra over $C$, and it is intuitively clear that the full quiver is the single path

$$
I \stackrel{\xi_{1} \alpha}{\longrightarrow} I I \stackrel{\xi_{2} \beta}{\longrightarrow} I I I
$$

$$
\left\{\left(\begin{array}{ccc}
C & \xi_{1} C & 0 \\
0 & C & \xi_{2} C \\
0 & 0 & C
\end{array}\right)\right\} .
$$

Conversely, suppose our algebra is representable over a commutative affine $F$ algebra $C$. Since $C$ is representable we can represent our algebra over $F$, but at the expense of a more complicated full quiver.

We turn to examples of permuted gluing:

Example 5.19. The branches

$$
I \rightarrow I \rightarrow I I \rightarrow I, \quad I \rightarrow I I \rightarrow I \rightarrow I
$$


are not glued, since the arrows are not labelled. On the other hand, the gluing between the branches

$$
\begin{aligned}
& I \stackrel{\alpha}{\longrightarrow} I \stackrel{\beta}{\longrightarrow} I I \stackrel{\gamma}{\longrightarrow} I, \\
& I \stackrel{\beta}{\longrightarrow} I I \stackrel{\gamma}{\longrightarrow} I \stackrel{\alpha}{\longrightarrow} I
\end{aligned}
$$

is permuted.

Likewise, the gluing between the branches

$$
\begin{aligned}
& I \stackrel{\alpha}{\longrightarrow} I I \stackrel{\beta}{\longrightarrow} I \stackrel{\gamma}{\longrightarrow} I I, \\
& I \stackrel{\gamma}{\longrightarrow} I I \stackrel{\beta}{\longrightarrow} I \stackrel{\alpha}{\longrightarrow} I I
\end{aligned}
$$

is permuted.

Note that the presence of permuted gluing dictates certain gluing of vertices (namely, the vertices corresponding to the glued arrows). We say that permuted gluing is complete if each arrow is glued to an initial arrow of (at least one) branch. (Here, by initial arrow, we mean the first arrow in the branch.)

We can characterize the full quiver with complete gluing. In order to avoid the trivial situation of a single arrow (so that the full quiver has length 1 ), we restrict our attention to length at least 2 .

Proposition 5.20. Any full quiver (of length > 1) with complete gluing of $m$ primitive arrows is a sub-quiver of a cube, of dimension $k \geq 2$, in which all the vertices are glued. For example, if there are three primitive arrows, the full quiver has the form

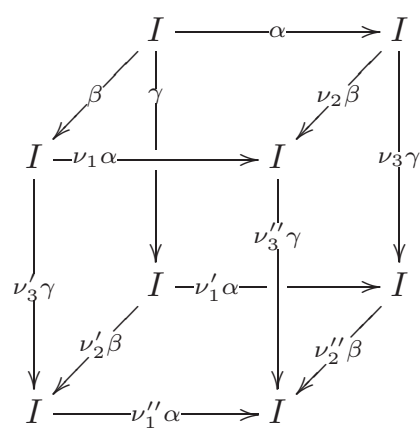

Proof. First of all, the assumption of complete gluing means that every arrow starts with a vertex labelled I, which means that all vertices except the last are glued to a vertex labelled I. But then the hypothesis of length $>1$ implies the last vertex also is glued to the vertex $\mathrm{I}$, since its arrow is glued to an arrow $I \rightarrow I$.

It remains to observe that all possible branches can be described inside the cube such that each arrow from the initial vertex is labelled $\alpha, \beta, \gamma$, etc., and all other arrows are proportionally glued. (This can be seen inductively by projecting onto a face of the cube.)

Example 5.21. Perhaps the most well-known example of permuted gluing is given by the Grassmann algebra $G=\sum_{i=1}^{n} F e_{i}$ on $n$ generators, defined by the relations 
$e_{i}^{2}=0$ and $e_{i} e_{j}=-e_{j} e_{i}$. $G$ has no nontrivial idempotents, so all vertices are glued. We start with two generators:

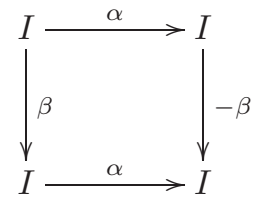

Likewise, one has the full quiver of the Grassmann algebra without 1, on two generators:

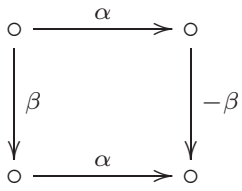

For the variety determined by the 3-generated Grassmann algebra (with unit element), the full quiver is the following cube:

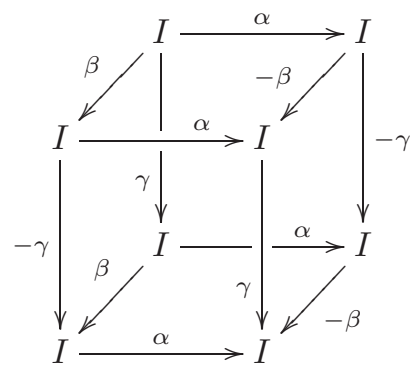

Analogously, the full quiver for the $m$-generated Grassmann algebra is the corresponding $m$-dimensional unit cube, with the analogous permuted gluing. (Although the full quiver can be nicely projected onto the plane for $m=3$, this becomes impossible for larger $m$.)

Here is an interesting related example.

Example 5.22. Let us consider the full quiver of the Grassmann envelope $E$ of the superalgebra $A=\mathbb{M}_{n, k}$, which is defined as $E=G_{0} \otimes A_{0} \oplus G_{1} \otimes A_{1}$, where the Grassmann algebra $G=G_{0} \oplus G_{1}$, and $A_{0}$ is the sum of the diagonal $n \times n$ and $k \times k$ blocks, whereas $A_{1}$ is the sum of the off-diagonal $n \times k$ and $k \times n$ blocks. The full quiver of the $m$-generated Grassmann envelope of the superalgebra $\mathbb{M}_{n, k}$ looks like two copies of the double of the full quiver of $G$, and may be described as follows:

Ordered pairs of vertices denoting blocks of size $n$ and $k$ respectively are located at each corner of the $m$-dimensional cube. We color the first component of each pair as red, and the second component as blue. In other words, the red components correspond to blocks of size $n$ and are glued among themselves, whereas the blue components correspond to blocks of size $k$ and are glued among themselves. These vertices correspond to the idempotents of $G_{0} \otimes A_{0}$.

The arrows correspond to elements of $G_{1} \otimes A_{1}$ and occur in pairs (in analogy to the full quiver of $G$ ). For each pair, one arrow connects the red component of the first vertex to the blue component of the second vertex, and another connects the blue component of the first vertex to the red component of the second vertex. 
Thus, there are two components, each of which looks like $G$. Each component corresponds to the same variety, but this description corresponds to the canonical representation of the superalgebra.

For example when there are $m=2$ generators, the full quiver of $A=\mathbb{M}_{n, k}$ is
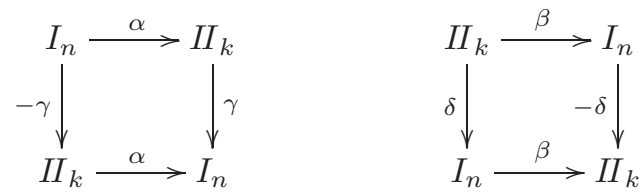

\section{IMPROVING THE FULL QUIVER}

In this section, we introduce techniques which enable us to choose the representation for which the full quiver has the "best" form. This quest, which is difficult enough when the base field is infinite, requires special techniques when the base field is finite.

6.1. Irreducible varieties. By quasi-linear variety, we mean an affine variety defined by quasi-linear equations. (Thus, for $F$ infinite, quasi-linear varieties are defined by linear equations.)

Lemma 6.1. Any quasi-linear variety $V$ over a finite field $F$ is the direct sum of the connected component $V_{0}$ of 0 , which is irreducible, and a space $V_{1}$ which is finite dimensional over $F$ and thus finite.

Proof. $V / V_{0}$ is a finite set, and also a vector space over $F$, and thus has some finite base $\bar{b}_{1}, \ldots, \bar{b}_{k}$, which we lift back to $b_{1}, \ldots, b_{k} \in V . V_{1}=\sum F b_{i}$ is annihilated by $p=\operatorname{char} F$ and is a subvariety since $V$ is quasi-linear, so we have split $V$ as $V_{0} \oplus V_{1}$.

We also need the following well-known fact from affine geometry:

Remark 6.2. Suppose $V$ is an irreducible affine variety. Then the coordinate algebra $\mathcal{O}$ is the projective limit $\lim \mathcal{O}_{s} / I_{s}^{n}$, where $\mathcal{O}_{s}$ is the local ring of coordinate functions, and $I_{s}$ is the ideal of functions vanishing at $s$, where $s$ is any fixed point of $V$.

In order to treat all irreducible varieties at once, we work in the set of formal power series $F\left[\left[\lambda_{1}, \ldots, \lambda_{m}\right]\right]$ over a field $F$ with $|F|=q$.

Definition 6.3. A $q$-power series is a power series $f\left(\lambda_{1}, \ldots, \lambda_{m}\right)$ in finitely many commuting indeterminates $\lambda_{1}, \ldots, \lambda_{m}$, with $f$ having constant term 0 , and all of whose finite truncations are $q$-polynomials. The $q$-power series $f$ has order 1 in $\lambda_{i}$ if some monomial of $f$ has degree 1 in $\lambda_{i}$. We let $H$ denote the subset of $F\left[\left[\lambda_{1}, \ldots, \lambda_{m}\right]\right]$ consisting of all $q$-power series.

By definition,

$$
H=\left\{\sum_{i=1}^{m} \sum_{j=0}^{\infty} \alpha_{i j} \lambda_{i}^{q^{j}}: \alpha_{i j} \in F\right\},
$$

but, for example, $\lambda_{1} \lambda_{2} \notin H$. Thus, $f \in H$ when each monomial of $f$ is a $q^{t}$-power of some $\lambda_{i}$ (which may vary according to the choice of monomial); $f$ has order 1 in $\lambda_{i}$ when some $\alpha_{i 0} \neq 0$. 
Remark 6.4.

(1) If $f\left(\lambda_{1}, \ldots, \lambda_{m}\right), g\left(\lambda_{1}, \ldots, \lambda_{m}\right) \in H$, with $g$ of order $i$ in $\lambda_{i}$, then

$$
f\left(\lambda_{1}, \ldots, \lambda_{i-1}, g\left(\lambda_{1}, \ldots, \lambda_{m}\right), \lambda_{i+1}, \ldots, \lambda_{m}\right) \in H .
$$

(In other words, $H$ is closed under composition of power series, as are linear mappings.) Indeed, writing $g\left(\lambda_{1}, \ldots, \lambda_{m}\right)=\sum_{i=1}^{m} \sum_{j=0}^{\infty} \beta_{i j} \lambda_{i}^{q^{j}}$, we see that for any $q$-power $q^{\prime}$,

$$
g\left(\lambda_{1}, \ldots, \lambda_{m}\right)^{q^{\prime}}=\sum_{i=1}^{m} \sum_{j=0}^{\infty} \beta_{i j} \lambda_{i}^{q^{j} q^{\prime}},
$$

since $|F|=q$ and char $F=p$.

(2) If $g=\sum_{i=1}^{m} \sum_{j=0}^{\infty} \alpha_{i j} \lambda_{i}^{q^{j}} \in H$ and we take $\alpha_{i u} \neq 0$ minimal among all the nonzero coefficients of $g$, then

$$
g^{q^{-u}}=\sum_{i=1}^{m} \sum_{j=0}^{\infty} \alpha_{i j} \lambda_{i}^{q^{j-u}},
$$

which has order 1 in $\lambda_{i}$. Thus, we can always apply (11) with $g^{q^{-u}}$.

The following result is the key to studying relations arising from $q$-polynomials. We say that variables are independent if there are no weak Frobenius relations among them.

Proposition 6.5. Suppose $V_{0}$ is an irreducible quasi-linear affine variety (containing 0$)$. Then there exist independent variables $\lambda_{i_{1}}, \ldots, \lambda_{i_{s}}$, such that all other $\lambda_{i}$ can be expressed in terms of q-power series in $\lambda_{i_{1}}, \ldots, \lambda_{i_{s}}$.

(This generalizes the following fact: In any system of linear equations, one can renumber the indices such that $\lambda_{1}, \ldots, \lambda_{s}$ are independent, and the other $\lambda_{i}$ are linearly spanned by $\lambda_{1}, \ldots, \lambda_{s}$.)

Proof. When char $F=0$, then "quasi-linear" means "linear" and there is nothing to prove. Thus, we may assume that $\operatorname{char} F=p>0$, and we need to show that whenever we have a power series equation involving indeterminates $\lambda_{i}$ expressed as an element of $H$, that we can solve some indeterminate in terms of the others. In view of Remark 6.4(2), we may assume that this has order 1 in some $\lambda_{i}$. Thus we have

$$
\sum_{i=1}^{m} \sum_{j=0}^{\infty} \alpha_{i j} \lambda_{j}^{q^{j}}=0
$$

with $\alpha_{i, 0} \neq 0$; dividing through by $\alpha_{i, 0}$, we may assume that $\alpha_{i, 0}=1$. To ease notation, we assume that $i=1$. Now we have $\lambda_{1}=\sum_{j=1}^{\infty} \alpha_{j} \lambda_{1}^{q^{j}}+\sum_{i=2}^{m} \sum_{j=0}^{\infty} \alpha_{i j} \lambda_{j}^{q^{j}}$. Thus substituting the right side for $\lambda_{1}$ yields another $q$-power series in which we have increased the degree of $\lambda_{1}$ (and furthermore have not produced new monomials of lower total degree). Each stage produces a new power series in which the degree of $\lambda_{1}$ in the monomials keeps increasing, so continuing indefinitely yields an element of $\mathcal{O}=F\left(V_{0}\right)$ in which $\lambda_{1}$ no longer appears, as desired.

Note that the final argument does not apply in $H$ itself, as $\lambda_{1}$ cannot be eliminated from the relation $\lambda_{1}=\lambda_{1}^{q}$; indeed, this relation defines a reducible variety. 
6.2. Reduction to proportional Frobenius gluing. In general, any type of gluing can occur in a representation. Indeed let $S$ be any set of $q$-polynomials on the $m^{2}$ variables $\Lambda=\left(\lambda_{i j}\right)_{i, j=1, \ldots, m}$. Then

$$
B_{S}=\left\{\left(\begin{array}{cc}
\alpha I & \Lambda \\
0 & \alpha I
\end{array}\right): \alpha \in F, \forall f \in S: f(\Lambda)=0\right\}
$$

is a Zariski closed algebra whose radical satisfies the relations in $S$.

We aim for the result that one can always find a representation of a Zariski closed algebra (over a suitable field extension) whose off-diagonal gluing is a consequence of proportional Frobenius gluing. This only seems to be accessible when the base field $F$ is infinite, so we start with this case; afterwards, we obtain the analogous result for arbitrary $F$ in case the algebra is relatively free.

Remark 6.6. As explained in Subsection 2.1 the relations on any semisimple subalgebra are of $F$-Frobenius type.

6.2.1. The case when the base field $F$ is infinite. When $F$ is infinite, the relations are linear. Then we may partition the arrows to independent variables, in terms of which all the other variables can be expressed. Suppose $\gamma$ is a dependent arrow, satisfying a relation of the form $\gamma=\sum_{i=1}^{k} \theta_{i} \alpha_{i}$, where the $\alpha_{i}$ are independent, and $\theta_{i} \in F$.

Replace the arrow $\gamma$ by $k$ arrows, identically gluing the $k$ initial vertices and (separately) the $k$ terminal vertices, as well as the resulting $k$ copies of any incoming or outgoing arrow from these vertices. The new arrows become $\gamma_{1}, \ldots, \gamma_{k}$, with the relations $\gamma_{i}=\theta_{i} \alpha_{i}$, so we only have proportional Frobenius gluing.

If the arrow $\gamma$ in the original full quiver shares no vertex with other arrows representing the $\gamma_{i}$, then the new full quiver represents the same algebra as the original one. However in general the procedure does not work, as the following example illustrates.

Example 6.7. The path

$$
I \longrightarrow \alpha \rightarrow I \longrightarrow \beta \rightarrow I-\gamma \rightarrow I
$$

with the linear relation $\alpha=\beta+\gamma$ could be expanded to

$$
\begin{aligned}
& I-\beta \rightarrow I-\beta \rightarrow I-\gamma \rightarrow I, \\
& I \longrightarrow \beta \rightarrow I \longrightarrow \beta \rightarrow I-\gamma \rightarrow I
\end{aligned}
$$

or to

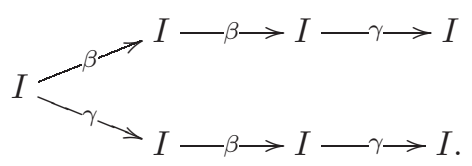

However in the original algebra, the generators $b$ and $c$ corresponding to the arrows marked $\beta$ and $\gamma$, respectively, satisfy the relation $b^{2}=c b$; this relation does not hold in the expanded algebras. The situation can be remedied by further 
identification; the full quiver (35) does represent the same algebra as the one in (32):

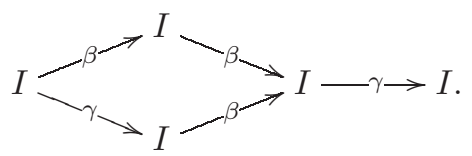

However, consider the same quiver, with $\alpha$ and $\gamma$ taken to be the independent variables, and $\beta=\alpha-\gamma$. The generators $a$ and $c$ corresponding to $\alpha$ and $\gamma$ satisfy $a^{2}+a c+c^{2}=0$. This relation does not hold for any intermediate expansion between (32) and

$$
\begin{aligned}
& I-\alpha \rightarrow I-\alpha \rightarrow I-\gamma \rightarrow I, \\
& I-\alpha \rightarrow I-\gamma \rightarrow I-\gamma \rightarrow I .
\end{aligned}
$$

A general algorithm would be rather intricate to describe, and is best done generically, motivating the proof of our next result.

Theorem 6.8. The Zariski closure of any representable affine PI-algebra A over an infinite field $F$ has a representation and full quiver all of whose polynomial relations are consequences of proportional gluing.

Proof. The radical $J$ of the Zariski closure $\hat{A}$ satisfies $J^{\ell}=0$ for some $\ell$, by Lemma 2.1. The proof is comprised of choosing basic linear functionals $\phi_{i}$ corresponding to the arrows of the original quiver. We write down the dependences of these arrows, and define a representation over a larger field in which we can obtain proportional relations involving the coefficients of these dependences, thereby producing a new quiver in which all of these dependences are described by proportional Frobenius gluing, which apply to the original linear functionals.

Since the base field $F$ is infinite, the Zariski closure $\hat{A}$ of the algebra $A$ is finite dimensional over $K$. Furthermore, all gluing among vertices is identical. We view $\hat{A} \subseteq \operatorname{End}_{K} V$, where $[V: K]=n$. Now we turn to the sub-Peirce decomposition of Remark 2.17, which is induced by the sums of idempotents along identically glued blocks. Some of the generators of $A$ belong to the semisimple component, and some to the radical component. We consider the finitely many sub-Peirce components of these radical elements, and their finitely many nonzero products (since all nonzero products have length $\leq \ell$ ).

Applying Gauss elimination to a basis of the space of linear dependences (as in [9. Corollary 6.5]), we may choose indices $i_{1}<i_{2}<\cdots<i_{m}$ such that there is no relation involving only the $\alpha_{i_{j}}$, while every other variable $\alpha_{u}$ can be expressed in their terms, via relations of the form

$$
\alpha_{u}=\sum_{j=1}^{m} \theta_{j, u} \alpha_{i_{j}}
$$

for $\theta_{j, u} \in F$.

Note that all such linear relations would be repeated in any glued vertices. Let $k\left(\alpha_{u}\right)$ denote the number of summands in (37). We call each such $\alpha_{u}$ a dispensable arrow; the arrows $\alpha_{i_{1}}, \ldots, \alpha_{i_{m}}$ are called indispensable. Note that we really are 
talking about linear functionals between the respective semisimple blocks, with their gluing, since any linear dependence in (37) must be repeated for every arrow glued to $\alpha_{i_{u}}$. Thus, strictly speaking, we are handling dispensable and indispensable linear functionals.

To form our extended full quiver, we start by taking any indispensable arrow $\alpha$ in the original full quiver, say from vertex $v$ to $w$, and replacing each with $k$ vertices $v^{(1)}, \ldots, v^{(k)}, w^{(1)}, \ldots, w^{(k)}$, and arrows from $v^{(i)}$ to $v^{(i)}$, all glued identically for each $1 \leq i \leq k$.

Thus, for the dispensable $\alpha_{i_{u}}$ in the original full quiver, say from $v_{i_{u}}$ to $w_{i_{u}}$, we put in $k\left(\alpha_{i_{u}}\right)$ classes of identically glued arrows, for each $i$ in the right side of (37). We observe that under the corresponding representation $A \mapsto \mathrm{M}_{n^{\prime}}(C)$ the gluing has become proportional. (In fact, most new gluing is identical; the only proportional gluing is between the $\alpha_{i}$ from the indispensable arrows and the $\bar{\theta}_{i} \alpha_{i}$ from the dispensable arrows, as illustrated in Example 6.11.)

The following example indicates just how far we can improve the full quiver.

Example 6.9. The proof of Theorem 6.8 separates the arrows of the representation

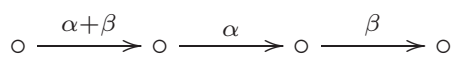

which are involved in nonproportional relations, to the form

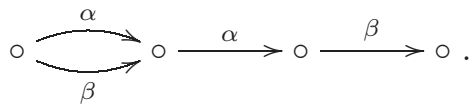

Note the presence of multiple edges to the left.

If one is willing to pass to relatively free algebras, one can further improve the full quiver. Since Theorem 6.8 permits us to reduce to the case that all gluing is proportional, we weaken Definition 5.15 slightly, and define an equivalence on arrows when they are proportionally glued; two branches are called proportionally permuted if for each arrow, the number of arrows proportionally glued to it is the same in each of the branches. (In other words, one branch is obtained from the other by permuting the arrows, but perhaps changing the proportionality constants in the gluing.)

Theorem 6.10. When $F$ is infinite, any relatively free affine PI-algebra $A$ has a representation whose polynomial relations are consequences of proportional gluing, and such that there are no double edges (between two adjacent vertices) in the full quiver. More generally, any two branches between two vertices are proportionally permuted.

Proof. The radical $J$ of the Zariski closure $\hat{A}$ satisfies $J^{\ell}=0$ for some $\ell$, by Lemma 2.1. The proof is comprised of choosing basic linear functionals $\phi_{i}$ corresponding to the arrows of the original quiver. We write down the dependences of these arrows, and define a representation over a larger field in which we can obtain 
proportional relations involving the coefficients of these dependences, thereby producing a new quiver in which all of these dependences are described by proportional Frobenius gluing, which apply to the original linear functionals.

We start as in the previous proof. Thus, applying Gauss elimination to a basis of the space of linear dependences (as in [9, Corollary 6.5]), we may choose indices $i_{1}<i_{2}<\cdots<i_{m}$ such that there is no relation involving only the $\alpha_{i_{j}}$, while every other variable $\alpha_{u}$ can be expressed in their terms, via relations of the form

$$
\alpha_{u}=\sum_{j=1}^{m} \theta_{j, u} \alpha_{i_{j}}
$$

for $\theta_{j, u} \in F$.

Note that all such linear relations would be repeated in any glued vertices. Let $k\left(\alpha_{u}\right)$ denote the number of summands in (40). We call each such $\alpha_{u}$ a dispensable arrow; the arrows $\alpha_{i_{1}}, \ldots, \alpha_{i_{m}}$ are called indispensable. Note that we really are talking about linear functionals between the respective semisimple blocks, with their gluing, since any linear dependence in (40) must be repeated for every arrow glued to $\alpha_{i_{u}}$. Thus, strictly speaking, we are handling dispensable and indispensable linear functionals.

Note that since the base field is infinite, $\hat{A} \otimes_{F} C$ is PI-equivalent to $\hat{A}$. Thus, we may work in $\hat{A} \otimes_{F} C$. We pass to the representation of $\hat{A}$ as transformations of $V^{\prime}=V \otimes_{F} C$, where the $\widehat{\theta_{i, u}}$ are commuting indeterminates over $F$ and $C=F\left[\widehat{\theta_{i, u}}\right] /\left\langle\widehat{\theta_{i, u}}\right\rangle^{\ell}$. (Since we have only finitely many $\theta_{i, u}$ needed for our finitely many relations, we see that $[C: F]<\infty$, and thus $\left[V^{\prime}: K\right]<\infty$.) Let $\bar{\theta}_{i, u}$ denote the canonical image of $\widehat{\theta_{i, u}}$ in $C$. Intuitively, the $\bar{\theta}_{i, u}$ behave like commuting indeterminates, except that the product of $\ell$ of these $\bar{\theta}_{i, u}$ is 0 . Thus the nonzero products $\tilde{\theta}$ of the $\bar{\theta}_{i, u}$ comprise a base $\Theta$ for $C$ over $F$; if $B$ is a base for $V$, then $B \otimes \Theta$ is a base for $V^{\prime}$ over $F$, of order $n^{\prime}=n[C: F]$.

To form our extended full quiver, we start by taking any indispensable arrow $\alpha$ in the original full quiver, say from vertex $v$ to $w$, and replacing it in the new quiver by arrows from $v \otimes \tilde{\theta}$ to $w \otimes \tilde{\theta}$, all glued identically for each $\tilde{\theta}$ in $\Theta$.

For the dispensable $\alpha_{i_{u}}$ in the original full quiver, say from $v_{i_{u}}$ to $w_{i_{u}}$, we put in $k\left(\alpha_{i_{u}}\right)$ classes of identically glued arrows; namely, for each $i$ in the right side of (40), we put in the identically glued arrows $\bar{\theta}_{i} \alpha_{i}$ from $v_{i_{u}} \otimes \tilde{\theta}$ to $w_{i_{u}} \otimes$ $\bar{\theta}_{i} \tilde{\theta}$. We observe that under the corresponding representation $A \mapsto \mathrm{M}_{n^{\prime}}(C)$ the gluing has become proportional and the endpoints have been separated unless the branches are proportionally permuted. (In fact, most new gluing is identical; the only proportional gluing is between the $\alpha_{i}$ from the indispensable arrows and the $\bar{\theta}_{i} \alpha_{i}$ from the dispensable arrows, as illustrated in Example 6.11.)

Theorem 6.10 will be improved in [1], by the introduction of a natural grading on the algebra.

Example 6.11. Consider again the full quiver (32) of Example6.7. The nilpotence index is $\ell=4$, and in the single relation $\alpha=\beta+\gamma$; here $\beta$ and $\gamma$ are indispensable, and $\alpha$ is dispensable. We thus take $C=F\left[\theta_{1}, \theta_{2}\right] /\left\langle\theta_{1}, \theta_{2}\right\rangle^{4}$, with the natural basis 
$\left\{\theta_{1}^{r} \theta_{2}^{s}: r+s \leq 3\right\}$, of cardinality 10. The expanded full quiver is

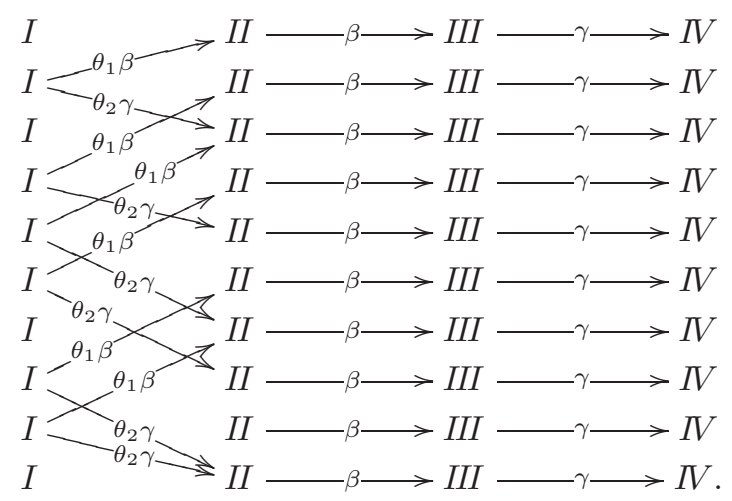

(The rows correspond to $\theta_{1}^{3}, \theta_{1}^{2}, \theta_{1}^{2} \theta_{2}, \theta_{1}, \theta_{1} \theta_{2}, 1, \theta_{1} \theta_{2}^{2}, \theta_{2}, \theta_{2}^{2}$ and $\theta_{2}^{3}$, in this order, for graphical reasons.)

The reduction to proportional Frobenius gluing has implications in the PI-theory. In [10, Example 6.1] we give an example of an algebra with parametric identities, which are defined over a commutative algebra extending of the base field $F$, but are not defined over $F$. However when we extend $F$ so as to obtain a representation with proportional gluing, we see that the parametric identities are now defined over the base ring.

One can see easily that identical gluing between branches yields parametric sets of identities. However, if the gluing is identity permuted, then the corresponding algebra does not have a parametric set of identities, since the proportionality coefficients are 1 . The details are to be given in [10.

6.2.2. The case of a finite base field. When the base field $F$ is finite, the situation is more intricate, since we have to deal with polynomial relations arising from Frobenius gluing. This forces us to deal with relatively free algebras.

Theorem 6.12. Any relatively free affine PI-algebra A has a representation for whose full quiver all gluing is Frobenius proportional.

Proof. We are done by Theorem 6.10 unless the base field $F$ is finite. Let $p=$ $\operatorname{char}(F)$. By [9, Theorem 7.10], the Zariski closure of $A$ has finite PI-rank, which means that it is PI-equivalent to a representable affine PI-algebra. Taking its generic elements, as constructed in [9, Construction 7.14] and studied in [9, Theorem 7.15], consider the algebra $\tilde{A}$ generated by these generic elements and pass to its Zariski closure $\hat{A}$.

In view of Lemma 6.1, we can decompose $J=V_{0} \oplus V_{1}$ as a direct sum of the connected component $V_{0}$ of 0 and a space $V_{1}$ which is finite dimensional over $F$ and thus finite, say with base $b_{1}, \ldots, b_{m}$. We take $\tilde{A}$ generated by $V_{0}$ and all elements $w_{k}=\sum_{j} \psi_{j, k} b_{j}$ of $V_{1}, 1 \leq k \leq m$, where $\psi_{j, k} \in F$. Note that $\psi_{j, k}^{q}=\psi_{j, k}$ since $|F|=q$. Then any element of $\tilde{A}$ can be represented as a linear combination of products of length $\leq \ell m$ of these elements. We need to replace the $w_{k}$ by generic elements $\sum_{j} \overline{\psi_{j, k}} b_{j}$ of $V_{1}$, where $\overline{\psi_{j, k}}$ are generic coefficients satisfying $\overline{\psi_{j, k}} q=\overline{\psi_{j, k}}$. These elements do not belong to $F$, but rather to the finite dimensional extension $F\left[\widehat{\psi_{j, k}}\right] /\left\langle{\widehat{\psi_{j, k}}}^{q}-\widehat{\psi_{j, k}}\right\rangle$, where $\widehat{\psi_{j, k}}$ are commuting indeterminates over $F$. 
We project onto the variety $\bar{V}$ defined by $q$-polynomials of degree $\leq q^{\ell m}$. Since the projection of a quasi-linear variety is quasi-linear, we may work in $\bar{V}$, in which all our $q$-power series project to $q$-polynomials. In view of Proposition 6.5, there is some subset of the arrows which is independent, and all other arrows can be written as $q$-polynomials in terms of these arrows.

Again we pass to the representation of $\hat{A}$ over

$$
\left(J \otimes_{F} F\left[\theta_{i, j}\right] /\left\langle\theta_{i, j}\right\rangle^{\ell}\right) \oplus\left(F\left[\widehat{\psi_{j, k}}\right] /\left\langle{\widehat{\psi_{j, k}}}^{q}-\widehat{\psi_{j, k}}\right\rangle\right)
$$

we can eliminate arrows as in the case for $F$ infinite (by the same technique of expanding the graph and labelling one arrow of each relation as "dispensable"), but this time our relations are in terms of $q$-polynomials rather than linear combinations. Thus, in this case, we can only reduce to proportional Frobenius gluing.

Note that Theorem 6.12 applies to $A$ as an algebra over the base field $F$, but not as a $K$-algebra. One can often extend Theorem 6.12 to reduce to the case that there are no double edges (between two adjacent vertices); this issue is to be treated in 10 .

This theory of full quivers (especially Theorem 6.12) enables us to characterize Noetherian properties of relatively free algebras, to be discussed in [10].

\section{Decomposing QUIVERS}

Let $A \subseteq \mathrm{M}_{n}(K)$ be an algebra in Wedderburn block form, and let $\Gamma$ be its full quiver. We turn now to the question of whether the decomposition of the full quiver $\Gamma$ has a matching decomposition of the algebra $A$ as a subdirect product. In [9] we discuss fine decompositions of $A$ related to various sets of idempotents in $A$. To every set of vertices $\Gamma_{0} \subseteq \Gamma$ there is an associated idempotent $e=e\left(\Gamma_{0}\right)$, which is the sum of the idempotents in $\mathrm{M}_{n}(K)$ corresponding to the matrix blocks of vertices in $\Gamma_{0}$. However, in general, $e$ is not an element of $A$ because of gluing (e.g., $e_{11}$ is not an element of $B_{\ell}$ in Example 5.4); nevertheless, we consider such idempotents, ignoring gluing altogether.

7.1. Decomposing quivers. We say that a quiver $\Gamma_{0}$ is convex if for every $i, k \in$ $\Gamma_{0}$, if $i \rightarrow j_{1} \rightarrow \cdots \rightarrow j_{t} \rightarrow k$ is a path connecting $i$ and $k$ in $\Gamma_{0}$, then $j_{1}, \ldots, j_{t} \in \Gamma_{0}$.

Proposition 7.1. If $\Gamma_{0}$ is convex, with $e=e\left(\Gamma_{0}\right)$, then $a \mapsto$ eae is a projection of algebras $A \rightarrow e A e$ (without 1). (Indeed, writing $a=\sum e_{i j} a_{i j}$ and $b=\sum e_{i j} b_{i j}$, convexity implies that eaebe $=$ eabe.)

Corollary 7.2. If every vertex of $\Gamma$ belongs to one of the convex sub-quivers $\Gamma_{1}, \ldots, \Gamma_{r}$ (which are not necessarily disjoint), then $A$ is a subdirect product of the algebras $A_{i}$ corresponding to the $\Gamma_{i}$.

Proof. The map $A \rightarrow A_{1} \times \cdots \times A_{r}$ is an algebra homomorphism by convexity, and it is injective by the covering assumption.

Example 7.3. Suppose the quiver contains three vertices with arrows glued in the form

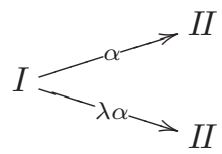

or

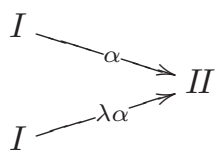


for some $\lambda \in K$. (The vertices must be glued, in view of Proposition 3.11). Then we can trade two arrows for one, by creating a new vertex, replacing the existing arrows with $I \stackrel{(\lambda+1) \alpha}{\longrightarrow} I I$.

To break down the full quiver further, we need another definition.

Definition 7.4. A pair of arrows is separated if the glued components of the two arrows are disjoint; i.e., there is no single branch that contains arrows glued to each of them.

A geometric decomposition $\Gamma \rightarrow \bigcup \Gamma_{i}$ of basic full quivers is a union of subquivers such that, for any arrow of $\Gamma_{i}$, all arrows of $\Gamma$ glued to this arrow also appear in $\Gamma_{i}$.

A full quiver is geometrically decomposable if it admits a proper geometric decomposition, and geometrically indecomposable otherwise.

Remark 7.5. Any full quiver with a pair of separated arrows is geometrically decomposable.

We would like the geometric decomposition of quivers to match a "geometric" subdirect decomposition of algebras. However, we are confronted with the following sort of example:

Example 7.6. Consider the algebra $A$ having full quiver $\Gamma$ given by

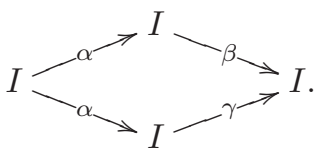

The geometric decomposition of $\Gamma$ is the union of the two full quivers

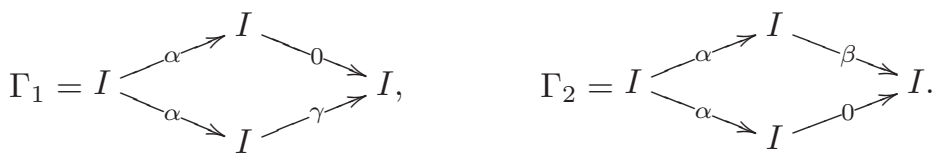

On the other hand, the subalgebra of $A$ corresponding to the full quiver

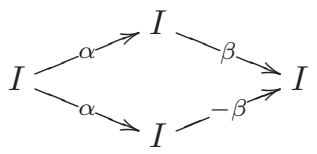

has the property that the radical squared is 0 , which fails in each of the algebras $A_{1}, A_{2}$ of (43), and it follows that $A$ cannot be a subdirect product of $A_{1}$ and $A_{2}$ as algebras.

Nevertheless, the algebra $A$ of Example 7.6 is a subdirect product of $A_{1}$ and $A_{2}$ as vector spaces, and the following observation is immediate:

Remark 7.7. If the full quiver $\Gamma$ of an algebra $A$ has a pair of separated arrows, then $A$ has subalgebras $A_{i}$ corresponding to the $\Gamma_{i}$ in the geometric decomposition of $\Gamma$, such that $A$ is a subdirect product of the $A_{i}$ as vector spaces.

Also, note that the obstruction in Example 7.6 to obtaining $A$ as a subdirect product of algebras came from a subalgebra of $A$ whose full quiver has extra gluing, 
which would not occur from elements in "generic" position. Thus, we will pursue these decompositions when studying polynomial identities in [10, 11].

Remark 7.5 can be refined further using Lemma 5.16. Let us embellish this discussion with a few more examples.

Example 7.8. (1) Consider the Grassmann algebra with two generators whose full quiver is given in (28). Since we are interested in its radical $J$, which is the algebra without 1 , we use the full quiver (29). Let $J_{1}$ be the subspace corresponding to the arrows $\alpha$, and $J_{2}$ be the subspace corresponding to the arrows $\beta$. There are also two arrows (denoted $\gamma$ ) corresponding to paths of length 2 , which correspond to a space $J_{3}$ :
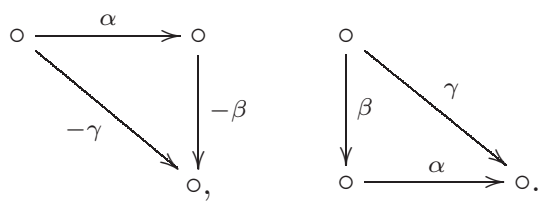

Thus, $J_{1}, J_{2}$, and $J_{3}$ correspond respectively to the operator spaces
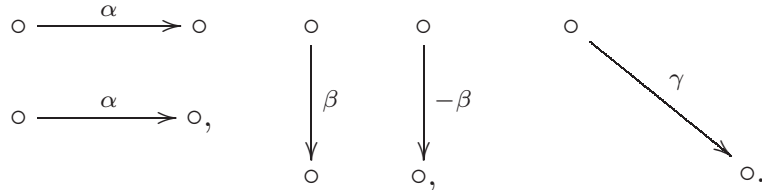

(1) The path

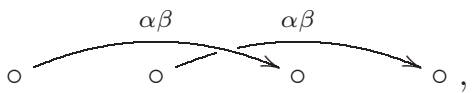

$\circ$

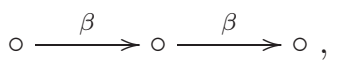
$\circ \stackrel{\alpha}{\longrightarrow} \circ$
$\circ \stackrel{\alpha}{\longrightarrow} 0$,
$\circ$

(s)

and the composition of two arrows (corresponding to $J^{2}$ ) is

noting that $\alpha^{2}=0$. The composition of three arrows (corresponding to $J^{3}$ ) is

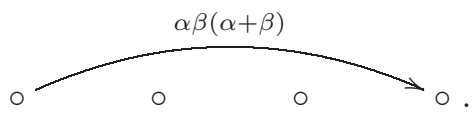

Remark 7.9. This leads us to the question of how to "restore" the imprimitive arrows in the full quiver. The answer is obtained by considering the full quiver of powers of the radical. More generally, if $A$ is an algebra without 1, we call an arrow pared if both of its vertices are $\circ$. Then any path $\circ \rightarrow \circ \rightarrow \cdots \rightarrow \circ$ of length $\ell$ comprised entirely of pared arrows corresponds to a primitive (pared) arrow in $A^{\ell}$. (This only works for pared arrows, since an element of $J$ matching an arrow having a vertex corresponding to an idempotent of $A$ can be multiplied as many times as one wants by that idempotent and remain the same unpared arrow of $A^{\ell}$ for each 
$\ell$. Thus, the full quiver of $A^{\ell}$ is comprised of all unpared arrows of the full quiver of $A$, as well as paths of length $\ell$ of pared arrows.)

For example, if $A$ is the algebra whose full quiver is

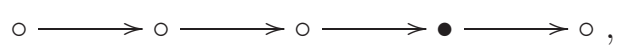

then the longest path of pared arrows has length 3 . Here $A^{2}$ corresponds to the sub-quiver

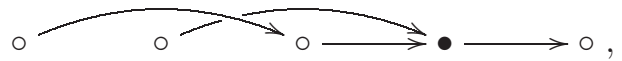

and $A^{n}$ (for every $n \geq 3$ ) correspond to the sub-quiver

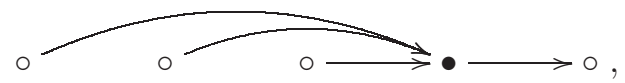

while $J, J^{2}$ and $J^{3}$ correspond to

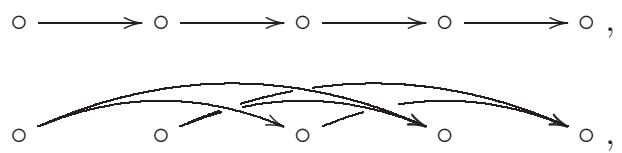

and

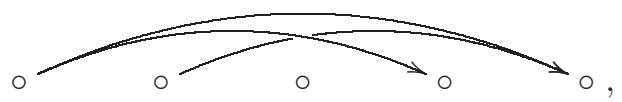

respectively.

\subsection{Idempotents and sub-quivers.}

Remark 7.10. Let $\Gamma_{0} \subseteq \Gamma$ and $e=e\left(\Gamma_{0}\right)$. Then $e A e$ is a subalgebra of $\mathrm{M}_{n}(K)$, whose full quiver is $\Gamma_{0}$. The relations of $e A e$ can be obtained from those of $A$ by removing all coefficients corresponding to vertices not in $\Gamma_{0}$. (We emphasize again that in general $e A e$ is not a subalgebra of $A$.)

The obstacles at the algebra level for "improving" quivers can be overcome only at the generic level, as we see in the next two examples.

Example 7.11. Consider the Zariski closed algebra $A$ whose full quiver is

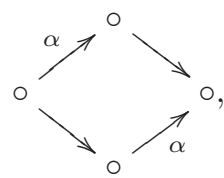

which is geometrically decomposed into two paths. Taking $x=e_{12}+e_{34}, y=e_{13}$ and $z=e_{24}$ we obtain the presentation $A=K\left[x, y, z \mid x^{2}=x y=y^{2}=y z=z x=\right.$ $\left.z y=z^{2}=0, y x=x z\right]$ (as an algebra without unit), and one easily checks that in any nontrivial quotient, $y x=0$ (which, incidentally, can be described in the same quiver, by imposing the relation that the imprimitive diagonal arrow is zero). Therefore, this algebra $A$ is subdirectly irreducible.

Example 7.12. Continuing with the algebra of Example [7.11, the algebra of generic elements has the presentation $\hat{A}=K\left[x, y, z \mid x^{2}=x y=y^{2}=y z=z x=\right.$ 
$\left.z y=z^{2}=0\right]$; namely, the relation $y x=x z$ no longer holds, and the quiver separates to

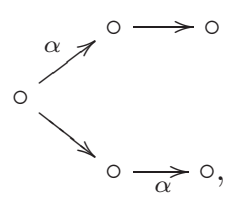

which has a geometric decomposition into two paths.

7.3. Appendix: Pseudo-quivers. Change of basis within a glued component can simplify the full quiver of the representation. We give a few examples and explain how they lead us to the notion of a pseudo-quiver.

Example 7.13. Consider the full quivers

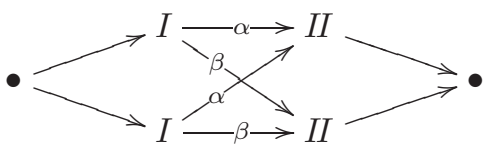

and

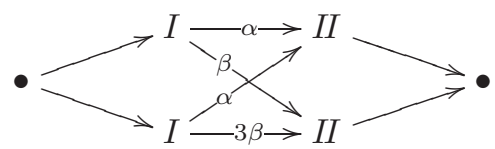

In the left-hand side of (53), a base change of the components denoted by $I$, which replaces $e_{22}, e_{33}$ with $e_{22}, e_{33}-e_{22}$ along the lines of Example 7.3 results in the full quiver to the left of (54), which is subdirectly reducible, with no gluing in each component. However in the right-hand side of (53), the same base change results in the quiver to the right of (54), which still has gluing.
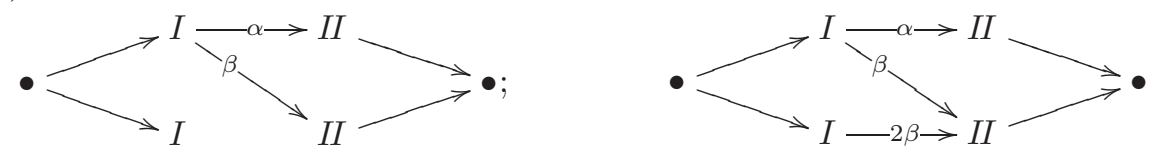

Example 7.14. Now consider the full quiver

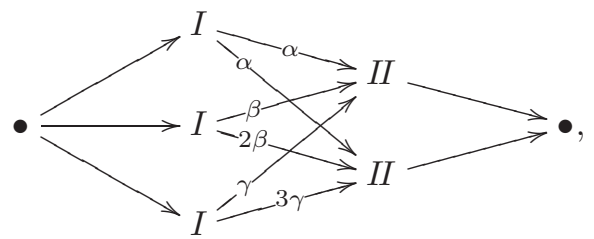

in which three pairs of arrows may be traded along the lines of Example 7.3, If not for the gluing, we could perform a base change resulting in the full quiver

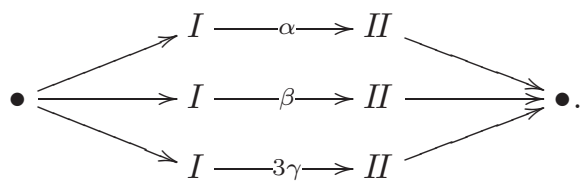

However the identical gluing of the $I$ components carries over to a linear dependence among the vertices denoted by $I I$, because otherwise the algebra would degenerate to the zero algebra. 
Recall that in a representation in Wedderburn block form, the diagonal blocks are direct sums of matrix algebras (over extensions of $F$ ). Gluing replaces the natural central idempotents of the glued blocks by their sum, and specifies a 'glued' subspace of the direct sum, such as $F(1,1,1) \subseteq F \oplus F \oplus F$.

Without diagonal gluing, a base change may simplify the arrows in the full quiver in a way that reduces the number of arrows. However such a base change may not preserve the diagonal gluing, resulting in a representation in which the diagonal blocks corresponding to vertices may have extra linear relations. Such a representation, which is no longer in Wedderburn block form, can be encoded in a full quiver with quasi-linear gluing among the vertices, which we call a pseudoquiver.

Let us see how we can improve the form of the pseudo-quiver by means of starting with the 'correct' representation.

Proposition 7.15. Suppose all off-diagonal gluing of the pseudo-quiver of a Zariski closed algebra is proportional. Then the algebra has a representation whose pseudoquiver (with respect to a suitable basis) has no linear dependence relations among arrows all starting or ending at the same vertex.

Proof. For any $a \in A$, we define its level to be the smallest $k$ for which $a J^{k}=0$. Suppose $m$ is the nilpotence index of $J$; i.e., $J^{m}=0$ but $J^{m-1} \neq 0$. We take a base of the functionals corresponding to arrows, starting with the top level $m-1$, and continuing inductively (which is possible, since the graph of the pseudo-quiver has no cycles).

View the arrows from the vertex $v$ as linear functionals. By definition, different arrows originating from the same vertex correspond to independent linear functionals. In this way, we see that any purely proportionally glued arrows originating from the vertex $v$ correspond to the same functional, and thus can be combined to a single arrow after a linear change of basis. The dual argument then applies to two arrows both ending at the same vertex. In this manner, we can eliminate all linear dependence relations among the arrows.

Definition 7.16. Making an open sandwich is the process of starting with the vertex $v$ and applying arrows corresponding to base elements of $J$, viewed as linear functionals as in the proof.

Corollary 7.17. When the base field $F$ is infinite, every algebra $A$ has a representation in which the linear functionals corresponding to the arrows of their pseudo-quivers are linearly independent.

Proof. We may start with a representation in whose pseudo-quiver the relations among the arrows are given by linear dependences, in view of [9, Proposition 4.7.(2)] (noting that quasi-linear relations are linear since $F$ is infinite). By Theorem 6.8, we have a representation whose pseudo-quiver can only have purely proportional gluing, which we eliminate using Proposition 7.15]

In view of Proposition 7.15, when studying relatively free algebras, we restrict our attention to pseudo-quivers that have only proportional Frobenius gluing among the arrows, and the only possible gluing among vertices comes from quasi-linear relations. In view of Corollary [7.17, when $F$ is infinite, we can always find a representation whose pseudo-quiver never has gluing from different arrows emerging from the same vertex (or, dually, leading to the same vertex). 
Although Corollary 7.17 may seem to be a technical curiosity, sacrificing the independence of the blocks for the (perhaps less desirable) linear independence of the arrows, it turns out to be a key tool in combinatorial PI-theory. Here is an application to the general structure theory.

Theorem 7.18. Any Zariski closed algebra A has a representation for whose full quiver the maximal length of the branches equals the index of nilpotence of the radical minus 1 .

Proof. Let $J$ be the radical, and take $m$ such that $J^{m}=0$ but $J^{m-1} \neq 0$. Recalling from the proof of Proposition 7.15 that the arrows of the pseudo-quiver correspond to a base of linear functionals, we can make an open sandwich by choosing one branch and zeroing out the other branches; in this way we eliminate cancellation among different branches.

In order to pass from pseudo-quivers to quivers, we consider the representation giving rise to the pseudo-quiver and take its corresponding full quiver. Note that the length of the maximal path of the pseudo-quiver is the same as the length of the maximal path of our full quiver.

This proof works for pseudo-quivers, but not necessarily for quivers, since we need the linear independence of the arrows.

Example 7.19. The full quiver of the Grassmann algebra on two generators (cf. Example (7.8) is also its pseudo-quiver. The radical $J$ is $F e_{1}+F e_{2}$. One open sandwich which we get is $F e_{1} e_{2} \neq 0$.

On the other hand, in the algebra of (44), the only open sandwich is composed of $e_{1}$ and $e_{2}+e_{3}$, as $\beta$ maps $e_{2}+e_{3}$ to zero, and indeed in this algebra $J^{2}=0$.

As we shall see in [10, when testing whether a polynomial $f\left(x_{1}, \ldots, x_{d}\right)$ is a polynomial identity of an algebra $A$, one observes that the monomials of $f$ can be determined to be zero or nonzero on $A$ according to their evaluations on paths of a suitable pseudo-quiver, whereas the analogous assertion for full quivers fails. Also, pseudo-quivers naturally give rise to PIs, as is to be seen in [10].

\section{REFERENCES}

[1] Assem, I., Skowronski, A., and Simson, D., Elements of the Representation Theory of Associative Algebras, Techniques of Representation Theory Series: London Mathematical Society Student Texts (No. 65) (2010), Cambridge Univ. Press. MR2197389 (2006j:16020)

[2] Belov, A., Counterexamples to the Specht problem, Sb. Math. 191 (2000), pp. 329-340. MR:1773251 (2001g:16043)

[3] Belov, A., Algebras with polynomial identities, representations, and combinatorial methods, Doctoral dissertation (2001), Moscow State University.

[4] Belov, A., On varieties generated by a ring which is finite-dimensional over its centroid, Comm. Moscow Math. Soc., Russian Math. Surveys 62:2 (2006), pp. 379-400. MR2352369 (2008e:16019)

[5] Belov, A., The Gel'fand-Kirillov Dimension of relatively free associative algebras, Sbornik Math. 195 (2004), pp. 1703-1726. MR2138478(2006c:16041)

[6] Belov, A.Ya., The local finite basis property and local representability of varieties of associative rings, Isvestija RAN 74:1, (2010), pp. 1-126. MR2655238(2011e:16039)

[7] Belov, A. and Rowen, L.H., Computational Aspects of Polynomial Identities, Research Notes in Mathematics 9, AK Peters, 2005. MR2124127(2006b:16001)

[8] Belov-Kanel, A., Rowen, L.H., and Vishne, U., Normal bases of PI-algebras, Advances in Applied Mathematics, 37(3), 378-389, (2006). MR2261179(2007f:16054) 
[9] Belov-Kanel, A., Rowen, L.H., and Vishne, U., Structure of Zariski closed algebras, Trans. Amer. Math. Soc. 362(9), 4695-4734, (2010). MR2645047

[10] Belov-Kanel, A., Rowen, L.H., and Vishne, U., Application of full quivers of representations of algebras, to polynomial identities, preprint.

[11] Belov-Kanel, A., Rowen, L.H., and Vishne, U., Classification of full quivers of representations of algebras, in terms of their polynomial identities, in preparation.

[12] Bernštel̆n, I. N., Gelfand, I. M. and Ponomarev, V. A., Coxeter functors, and Gabriel's theorem (Russian) Uspehi Mat. Nauk 28(2(170)), 19-33, (1973). MR0393065 (52:13876)

[13] Gabriel, P., Unzerlegbare Darstellungen I., Manuscripta Math. 6, 71-103 (1972). MR0332887 $(48: 11212)$

[14] Giambruno, A. and Zaicev, M., Minimal varieties of algebras of exponential growth, Adv. Math. 174 (2003), pp. 310-323. MR1963697 (2004b:16028)

[15] Giambruno, A. and Zaicev, M., Polynomial identities and asymptotic methods (2006). MR2176105 (2006g:16054)

[16] Grishin, A.V., Examples of T-spaces and T-ideals in Characteristic 2 without the Finite Basis Property (in Russian), Fundam. Prikl. Mat. 5(1), no. 6, 101-118 (1999). MR.1799541 (2002a:16028)

[17] Kac, V., Infinite root systems, representations of graphs, and invariant theory, Inv. Math. 56, 57-92, (1980). MR 557581 (82j:16050)

[18] Kac, V., Infinite root systems, representations of graphs, and invariant theory, J. Algebra 78, 141-162, (1982). MR677715 (85b:17003)

[19] Kambayashi, T., Miyanishi, M. and Takeuchi, M., Unipotent algebraic groups, Lecture Notes in Mathematics 414, Springer-Verlag, Berlin-New York, 1974. MR0376696 (51:12871)

[20] Kemer, A.R., The representability of reduced-free algebras, Algebra i Logika 27(3), 274-294, (1988). MR997959 (90e:16027)

[21] Kemer, A.R., Identities of Associative Algebras, Transl. Math. Monogr., 87, Amer. Math. Soc. (1991). MR1159223(93d:16027)

[22] Lewin, J., A matrix representation for associative algebras. I and II, Trans. Amer. Math. Soc. 188(2), 293-308, 309-317 (1974). MR0338081 (49:2848)

[23] Miyanishi, M., Questions of rationality of solvable algebraic groups over non-perfect fields, Annali Mat. Pura Appl. 61(4), 97-120, (1963). MR0158891(28:2113)

[24] Rowen, L.H., "Polynomial Identities in Ring Theory", Pure and Applied Math., 84, Academic Press, New York, 1980. MR576061(82a:16021)

[25] Rowen, L.H., "Ring Theory", AK Peters, 1988.

[26] Tits, J., Lectures on algebraic groups, Dept. of Math., Yale Univ. of New Haven, 1966/67.

Department of Mathematics, Bar-Ilan University, Ramat-Gan 52900, Israel

E-mail address: belova@macs.biu.ac.il

Department of Mathematics, Bar-Ilan University, Ramat-Gan 52900, Israel

E-mail address: rowen@macs.biu.ac.il

Department of Mathematics, Bar-Ilan University, Ramat-Gan 52900, Israel

E-mail address: vishne@macs.biu.ac.il 